\title{
INDOURALICA IX
}

Dieser Aufsatz ist der Frage gewidmet, was entschpricht auf der indogermanischen Seite den uralischen Klusilen - $p$ - oder - $t$-in intervokalischer Lage. Der Aufsatz war schon 1973 fertig, dann schickte ich ihn am 5. XII. 1973 dem Redakteur von Orbis, wo er laut einem brieflichen Versprochen von van Windekens im nächsten oder zweitnächsten Jahr (1975) erscheinen sollte; das wurde in einem Programm von Orbis in folgenden zwei Jahren zweimal bestätigt, danach verschwand jede Spur dieses Manuskriptes; anstatt dessen tauchten mehr und mehr zahlreiche. Invektiven des belgischen Sprachwissenschaftlers gegen den.Verfasser des vorliegenden Textes hervor. Da jede Auseinandersetzung mit van Windekens und seiner Methode nutzlos wäre, odi profanum vulgus et arceo, bemerke ich nur noch, dass mein Aufsatz in einer Kopie bei mir erhalten blieb, dass ich dieses Manuskript sofort an einen anderen Verleger liefern könnte, wurde jedoch wegen einer langwierigen Krankheit in meinem Schaffen so stark gehemmt, dass ich es erst in letzter Zeit in die Hände nehmen durfte; ich erkannte, dass zwar schon die alte Fassung genügte; da jedoch die zwar zehnjährige Verspätung doch einen so weiten Zeitraum bedeutete, dass immerhin einige Nachträge und Verbesserungen gemacht werden sollten. So entstand der vorliegende Text, der in der Methode und Ziel die Hauptlinien der ganzen IndouralicaReihe verfolgt. Wesentliche Veränderung bedeutet nur meine noch vor 1980 gefasste Regel, dass der ural. $\breve{s}$-Laut im Idg. zum Hauchlaut $* h$ wurde (mit einigen speziellen Abschwenkungen) und so entstand der Aufsatz Indouralica XIX, der bald dem Publikum zur Verfügung dargeboten werden soll.

Die Situation in der Veröffentlichung der IU-Reihe ist nun wie folgt:

I: ural. $m, n, l, r=$ idg. $m, n, l, r$, erschien als selbständige Arbeit bei der Slovenischen Akademie der Wissenschaften (= SAZU) II 30/1 (Ljubljana 1974), 120 Seiten;

II: ural. $j, w=$ idg. $i, u$ (oder $j, w)=$ UAJb.44 (1972), 162-178;

III: ural. Sibilanten $s, \hat{s}=$ idg. $s$, in Vorbereitung; s.XIX;

IV: ural. anlautende Tenues $=$ idg. anlautende Tenues (oder $s+$ Tenues), erschien in Linguistica XIII (Ljubljana 1973), 116-190;

V: ural. anlautende Tenues $=$ idg. anlautende Mediä aspiratä $=$ Collectanea Indo-Europaea I (Ljubljana 1978, in: Series comparativa III), 145-196;

VI: ural. Entsprechungen der indogermanischen an- und inlautenden Mediä $b$, $d, g, g, g u$, in Vorbereitung;

VII: ural. $\eta=$ idg. $g, n g=$ KZ.84 (1970), 151-174;

VIII: indogermanische Entsprechungen der uralischen starken (doppelten, langen) Tenues $p p, t t, k k$ (nur im Inlaut) = ALHung.24 (Budapest 1974), 87-116; 
IX: indogermanische Entsprechungen der uralischen inlautenden schwachen Tenues $p, t$; im Druck = der vorliegende Aufsatz);

$\mathrm{X}$ : indogermanische Entsprechungen der uralischen schwachen Tenuis $k$ und des Spiranten $\gamma=$ Lingu. XXV (Ljubljana 1985), 193-262;

XI: indogermanische Entsprechungen der uralischen inlautenden Lautgruppen vom Typus $l, r, m, s, s, c, \check{c},+k$ oder $\gamma$, in Vorbereitung;

XII: indogermanische Entsprechungen der uralischen inlautenden Lautgruppen vom Typus $p$ bzw. $k+$ Konsonant, im Druck;

XIII: indogermanische Entsprechungen der uralischen Affrikaten $c, \breve{c}$ im Anlaut; in Vorbereitung;

XIV: indogermanische Entsprechungen der uralischen inlautenden Affrikaten $c, \check{c}=$ Orbis XIX (1970), 282-323;

XV: indogermanische Entsprechungen der uralischen inlautenden Spiranten $\delta$ und $\delta^{\prime}=\mathrm{KZ} .87$ (1973), 41-58;

XVI: indogermanische Entsprechungen der uralischen Lautgruppen vom Typus Nasal + Verschlusslaut = Orbis XXII (1973), 5-42;

XVII: einfache Vokale auf beiden Seiten, im Druck;

XVIII: indogermanische Entsprechungen uralischer Verbindungen vom Typus $+j$ oder $w+$ Konsonant, in Vorbereitung;

XIX: ural. $\breve{s}=$ idg. $h$ (s. oben bei IU.III; als ich erkannte dass $\breve{s}$ ganz andere Wege ging als $s$ und $s$, machte ich daraus einen selbständigen Aufsatz), in Vorbereitung.

Anm.: Die Ergebnisse der Aufsätze X, XI, XII sind vorläufig in meiner Arbeit Die indouralische Sprachverwandtschaft und die indogermanische Laryngaltheorie, SAZU.VII/5 (Ljubljana 1970), 64 Seiten, erreichbar.

Wie schon oben bemerkt, ist der vorliegende Aufsatz der Frage gewidmet, wie die uralischen Klusilen $p$ und $t$ (im In- und Auslaut; für den Anlaut gilt der Aufsatz IV, s. oben) im Indogermanischen vertreten werden.

Das Uralische scheint einst im Besitz zweier Arten inlautender Klusilen gewesen zu sein:

a) st ar ker (doppelter, langer): $-p p-,-t t-,-k k-$ (IU.VIII);

b) schwacher (einfacher, kurzer): $-p-,-t-,-k-$.

Die ersteren bilden den Inhalt von Indouralica VIII; von den letzteren kommt $k$ in den Aufsätzen X-XII zur Sprache und ist schon ziemlich ausführlich auch in der Arbeit des Verf. Die indouralische Sprachverwandtschaft und die indogermanische Laryngaltheorie, Acad. Sc. et Art. Sl., Cl. II, VII/5, Ljubljana 1970, besprochen worden. Die vorliegende Untersuchung wird demnach absichtlich auf die inlautenden Laute $p$ und $t$ beschränkt, und zwar nur auf solche in intervokalischer Stellung. 
Ich habe während langjähriger Versuche und Überlegungen folgende Entsprechnungsregeln festgestellt:

a) dem uralichen schwachen inlautenden $p$ entspricht im Idg. der Halbvokal $\underset{\sim}{u}$;

b) dem uralischen schwachen inlautenden $t$ entspricht im Idg. gewöhnlich der Spirant $s$, nach Gutturalen jedoch $\boldsymbol{b}$.

Daneben kommt als Folge besonderer kombinatorischer Bedingungen noch idg. $d$ als Entsprechung des ural. $t$ in Frage, selten sogar $t$.

Da die in Betracht kommenden Wörter bzw. Wurzeln auch noch im Anlaut weitere Konsonanten aufweisen, ist es am Platze, auch darüber kurz zu berichten:

a) ural. $m=$ idg. $m$, ural. $n, n, n=$ idg. $n$; ural. $l, l, l=$ idg. $l$, ural. $r=$ idg. $r$;

b) ural. $s, s=$ idg. $s$; ural. $\breve{s}=$ idg. $h$;

c) ural. $j=$ idg. $i$, ural. $w=$ idg. $u$;

d) an la ut end ural. $p=$ idg. $p$ oder $s p$ oder $b h$; ural. $t=$ idg. $t$ oder $s t$ oder $d h$, ebenso die ural. Affrikatä $c, \check{c} ;$ ural. $k=$ idg. $k, q, q^{w}$ oder $s k$ usw. oder $g h$ usw.;

e) ural. Vo kal e der ersten Silbe: $a, o, u=$ idg. (gewöhnlich) $a$ oder (sekundär) $e$; ural. $\ddot{a}, e, i, \ddot{u} ; e, \underline{i}=$ idg. $e$.

Spezielle Entsprechungen werden an Ort und Stelle näher behandelt. Hier erwähne ich noch, dass als Entsprechung ural. $\breve{c}$ im Anlaut oft idg. $d$-vorkommt.

\section{Literatur (nur in Auswahl):}

J. Szinnyei, Finnisch-ugrische Sprachwissenschaft, 2. Aufl., Berlin und Leipzig 1922 (= Szinnyei $\left.{ }^{2}\right)$; elvh. ${ }^{7}$ );

J. Szinnyei, Magyar Nyelvhasonlítás, 7. Aufl., Budapest 1927 ( = Szinnyei, Ny-

Bj. Collinder, Fenno-Ugric Vocabulary, Stockholm 1955 (= Collinder I); 2. Auflage Hamburg 1977;

Bj. Collinder, Comparative Grammar of the Uralic Languages, Stockholm 1960 ( = Collinder III);

Bj. Collinder, Indo-uralisches Sprachgut, Uppsala 1934 (= Collinder, IU.);

Bj. Collinder, Hat das Uralische Verwandte?, Uppsala 1965 (= Collinder, U.);

Y. H. Toivonen (E. Itkonen, A. J. Joki), Suomen kielen etymologinen sanakirja, Helsinki 1955ff. (= Toivonen; oder SKES.);

V. I. Lytkin, E. S. Guljajev, Kratkij etimologičeskij slovaŕ komi jazyka, Moskva 1970 ( = Lytkin-Guljajev);

G. Bárczi, Magyar szófejtő szótár, Budapest 1941 (= Bárczi); 1976.

A Magyar Nyelv Történeti-etimológiai Szótára, I Budapest 1967, II 1970, III

B. Čop, Die indogermanische Deklination im Lichte der indouralischen vergleichenden Grammatik, SAZU. II/31, Ljubljana 1975 (= Čop, Dekl.);

B. Cop, Die indouralische Sprachverwandtschaft und die indogermanische Laryngaltheorie, SAZU. Diss. VII/5, Ljubljana 1970 (= Čop, Laryng. 1970);

B. Čop, IU. = B. Čop, Indouralica (19 aufsätze bzw. selbständige Hefte = I-XIX, s. oben in der Einleitung); darunter IU. I (ural. $m . n, l, r$ ), erschein als selbständige Arbeit bei SAZU. II 30/1 (Ljubljana 1974; s. oben in dem conspectus der Reihe); 
T. Lehtisalo, Über die primären ururalischen Ableitungssuffixe, Helsinki 1936, (= Lehtisalo);

Finnisch-ugrische Forschungen 1ff. = 1901ff., Helsinki; = FUF;

Sovetskoje finno-ugrovedenije, Tallin 1965ff. (= SFU.);

Ural-Altaische Jahrbücher, Wiesbaden (= UAJb.).

\section{Zum Nostratischen:}

V. M. Illič-Svityč, Opyt sravnenija nostratičeskih jazykov I: Moskva 1971; II 1976 ( = Illič-Svityč).

Manche Vergleichung auf diesem Gebiete findet sich auch in meinen Arbeiten, so: UAJb. 44 (1972), S. 289;

Orbis XIX (1970), SS. 314-321;

Laryng. 1970, SS. 218/36-219/37;

Orbis XXII (1973), SS. 39-41;

KZ. 84, (1970), S. 159 und 162-163 Anm. 13;

KZ. 88 (1974), SS. 46;

Linguistica XIII (1973), SS. 173-189;

AlHung. 24 (1974), SS. 114-115;

Coll. Ieur. I (Ljubljana 1978), SS. 184-196;

Linguistica XXV (1985), SS. 245-247.

Aus einem verwandten Bereich stammt der Aufsatz: B. Čop, Méditerranéen et indo-ouralien (I.) (Ljubljana 1976) in Linguistica XVI, SS. 3-33.

Vgl. noch:

N. Poppe, Vergleichende Grammatik der altaischen Sprachen, Teil I, Wiesbaden 1960 (= Poppe I).

M. Räsänen, Uralaltaische Wortforschungen, Helsinki 1955 (= Räsänen). Viele derartige Etymologien bringt Collinder in $\mathrm{I}^{2}$ SS. 152-158; U., SS. 136-155.

Mein Versprechen, eine komplette indouralische Morphologie (samt Wortbildungslehre) zu schreiben, ist leider noch nicht erfüllt worden, mit Ausnahme von Dekl. (s. oben); doch ist darüber hinaus im II. Teil jeder. Arbeit aus der IU.-Reihe unter dem Titel Suffixgleichungen (wenn die Arbeit solches Material überhaupt liefert) auch solchen Elementen Platz geboten. Dasselbe gilt auch für Laryng. 1970 usw.

\section{Wortgleichungen}

Die uralischen Urwörter $=$ Wurzeln weisen gewöhnlich folgende Lautstrukt ur auf: $C V p V$ (oder ohne anlautenden Konsonanten); auf indogermanischem Bo- 
den ging der zweite Vokal durch die Lautreduktion oft verloren oder wenigstens wurde er nicht mehr als Wurzelbestandteil gefühlt; idg. Struktur demnach $C V C$ oder $V C$, wo der dem $V$ folgende Konsonant $=$ (das aus iu. $-p$ - entstandene) $-w$-, also $(C) V w$.

(1) finn. apu "Hilfe“, apu-lainen "Gehilfe“, au-tta-a „helfen, beistehen" < *aß-utta-, karel. olon. abu, autta-, estn. abi „Hilfe; Gehilfe“ usw., nur ostseefinn. (Toivonen = SKES. 22b):

$\sim$ idg. *au- „gern haben; verlangen; hilfreich sein, begünstigen“, *awes- „Hilfe". (Pokorny 77ff.), z.B. in ai. ávati "verlangt, begünstigt, fördert", av. avaiti „sorgt, hilft", air. con-ö $i$ „beschützt", ai. ávas- „Befriedigung, Gunst, Beistand“ = av. avah- „Hilfe“, ai. $\bar{o}$-man- „günstig, helfend" = av. a $\bar{o}-$-man- „helfend, beistehend“, ai. $\bar{u} t t^{\prime}$ „Förderung, Hilfe“, lat. aveō „begierig sein, heftiges Verlangen tragen“. Urspr. beiderseits "wohlwollend, huldvoll, begünstigend (sein)“. Das finn. Wort ist deverbal, auf $S x-u$.

Mit Nr. (29) ist unsere Übersicht am Ende; die in der Einleitung erwähnte erste Fassung dieses Aufsatzes ist jetzt um etwa 3/5 vermehrt worden; neu sind folgende Etymologien:

1. mit urspr. $-p-$ :

(3) gr. ou „nicht", (5) kepeä „leicht“, (7) kup- „Lunge“, (12) puhde Frühmorgenzeit“, (13) *s $\bar{a}-w$ - „funkelnd“, (16) *šäpä(-s) „Hals“, (17) kipinä „Funke“, (18) *upeš- „stallion, ram“, (19) korpe- „sengen, brennen“, (20) njarbâd „thin“;

2. mit urspr. $-t-$ :

Die Suffixgleichungen sind unverändert geblieben, obwohl einiges hinzuzufügen wäre. Ich komme darauf anderswo.

(2) zum uralischen Negationsverb * $e$ - ,nicht" (z.B. in finn. $e-n$,ich nicht...", $e-i$ „nicht", Collinder I 10) wurde in einigen f.-ugr. Sprachen ein Part. Präs. mittels des $S x$ *-pa-gebildet: $e$-pä „nicht seiend“ = finn. e-pä- „un-“" (z.B. epä-huomio „Unachtsamkeit" usw.), karel. olon. ebä-ds., estn. eba-, z.B. eba-jalg „Windwirbel“ = „Unfuss“ u.a., wotj. *eb- in *evel ,ist nicht“, syrj. *ab- usw. in $a b u$,ist nicht“, „es gibt nicht", urspr. *ab- + vel- „sein“ (Toivonen 40; Lytkin-Guljajev 29):

$\sim$ idg. *eu- "mangeln, leer" bei Pokorny 345f., z.B. in ai. $\bar{u}$-ná- und av. $\bar{u}-n a-$,,unzureichend, ermangelnd“, npers. vang „leer, arm“, arm. $u$-n-ain „leer“, gr. $\varepsilon \widetilde{J}-v \iota-s$,beraubt, ermangelnd“, got. $w a-n-s$ (usw.) „mangelnd, fehlend“, was alles wohl auf Part. Perf. Pass. auf -no-von einem denominalen Verbalstamm *ewa-H- „nicht seiend sein, fehlen, mangeln" (vom persönlichen Subjekt), „mit Nichtsein behaften, ermangelnd machen" fusst, ein je-Verb ohne laryngales $S x$ kann in av. $u-y a-$, nur. Part. uyamna-, „nicht ausreichend, ungenügend, mangelhaft" gesucht werden, $=* u$ u-je/o- direkt zu *eu- „nicht seiend“. 
Das Indogermanische kannte also auch negatives ${ }^{*} e$-, wodurch nun ein negativtypologisches Merkmal aus der Indouralistik entfernt wird. Verwandt ist Nr. (3); beide zum Part. auf * ${ }^{*} a-$, Kapitel II (2).

(3) es ist möglich, die bekannte gr. Negation *ou „nicht“ bei ur. Negationsverbum *e- "nicht“" (Collinder, FUV. 31 usw.) - s. oben Nr. (2) - anzuknüpfen; es ist im Griechischen seit ältesten Zeiten als Substitut des idg. *ne belegt, auch im Mykenisch (KN, PY) und immer als echter Diphthong /ou/geschrieben (Ausnahme einmal vor $w o-z e)$, auch verstärkt $o-u-k i$ und konjunktionell $o$ - $u$-qe ,und nicht, doch/aber nicht", vgl. Vilborg, Tent. Gr. 123f. und 128.

Wenn obige Etymologie richtig ist, hat man mit $o$-stufigem Stamm ${ }^{*} o$ - "nicht zu tun, woraus das iur. Partizip auf ${ }^{*} p e$ - gewonnen wurde (also ${ }^{*} o-p e>{ }^{*} o-u-$ mit Reduktion des auslautenden $-e$ ). In Betreff der dialektologischen Stellung stimmt unser Fall mit meinen Ausführungen in Heth. und Idg. (Innsbruck 1979), SS. 9-24.

(4) hierher gehört 'wohl auch finn. ilves (kse-Stamm) „Luchs“, karel. ilveš, olon. ilves, lüd. ilvez, weps. ilbez, estn. ilves, lapp. L alpas, $\mathrm{N}$ âlbâs ds., Urform also *ilpe-kse-, ohne weitere Beziehungen (Toivonen 106), ausser im Altaischen (Räsänen 14, vgl. osttürk. jilpis „Schneeleopard“, tel. irbis „eine Luchsart“ usw.):

$\sim$ idg. *lu-k-, *lu-n-k- „Luchs" in arm. lusanun-kh Pl., gr. $\lambda \cup \gamma \xi, \lambda \cup \gamma \varkappa \delta \varsigma$, ahd. luhs, ags. lox, aschwed. lo $<*$ luha-, lit. lúšsis usw. bei Pokorny 690. Die eventuelle Möglichkeit, dies Wort mit ai. rúsáant- „licht, hell, weiss“ zu verbinden, scheitert an der Bedeutung; wohl aber wird sl. rys6 sein $r$ - einer Volksetymologie verdanken, vgl. über solche Möglichkeiten Vasmer, Russ. EW. II 557.

Im Uralischen ist $-k s e$ - ein $S x$, das oft in Deminutiven u. ähnl. auftritt, darunter auch solchen, die von Tiernamen ausgehen (vgl. z.B. finn. koira-kse- „Männchen“ von koira „Hund" und Szinnyei ${ }^{2}$ 84f.); als Wortkern ist also ein *ilpe- anzunehmen. Im Indogermanischen ist das $-k$ - ein $S x$, das auch im Wort für "Fuchs" usw. auftritt (s. Pokorny 1179): ai. lōpāsá- „Schakal, Fuchs“, arm. aluēs „Fuchs“, gr. å $\lambda \omega \pi n \xi$ ds., lett. lapsa, endlich lit. vilpiš̃ys „wilde Katze“; zu anderweitigem Material Brugmann, Grdr., ${ }^{2}$ II 1, 479 und 484. Im Indogermanischen ist demanch als Wurzel

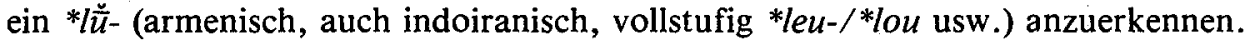

Mann wird also die Gleichung ural. *ilpe- = idg. *leu- aufstellen, wobei als urindoural. etwa dreisilbiges *ilepe- „Luchs“ anzunehmen ist. Ob nun hinter diesem Wortgebilde eventuell ein Farbelement *ile- steckt, das etwa mit idg. Farbwurzel *el- „rot, braun“ bei Pokorny 302ff. identisch wäre, sei dahingestellt, obwohl bedeutungsmässig der "Luchs" mit „der Rote" sehr gut bennant wäre und weiter ein $k$-Element auch in der Ableitung von *el- (u.a. *ól-k-i-s „Elch“ (s. Pokorny 303 unten) vorliegt.

Ansonsten kann die Verwandtschaft beider Wortsippen dadurch betont werden, dass man idg. ${ }^{*}-k$ - als mit dem ural. ${ }^{*}-k s e-$ historisch identisches Suffix ansieht. Mehr anderswo. 
(5) finn.-ugr. *kepe- $\delta \ddot{a}$ in finn. kepeä usw., auch keveä „leicht, opp. schwer, schwierig; u.a.“, dazu kevyt "leicht", ke-yhkä id., „flink, behend" ua., keuhko "Lunge“, lapp. N gâepâd "leicht", gâeppanit "sich vermindern, kleiner werden“ u.a., gâeppes „Lunge“, wotj. kap-tśi „leicht“, ung. kevés „wenig, gering“ (opp. "viel"); sam. jur. sibi, jen. sebi usw. "leicht" (Collinder, FUV. ${ }^{2} 44$; SKES. 182 usw.):

$\sim$ gr. koü-ph-os „leicht, von geringer Schwere, leichtbeweglich usw.“, seit Homer belegt und somit uralt, obwohl im Griechischen isoliert und sonst ohne gute Etymologie, s. Frisk, Gr. EW. I 936. In koũphos steckt eine sonst verlorene idg. Wurzel *(s)keu- (mit einer Variante auf ${ }^{*}-b h$-), die auf „geringe Schwere, leichte Tragbarkeit, Beweglichkeit, Ausführbarkeit“, auch „psychisches Schaffen“ (vgl. finn. kevyt-mielinen "leichtsinnig“ = gr. kouphó-noos ds.) auf beiden Sprachgebieten angewandt wurde.

(6) ural. *kopa "Borke, Rinde, Schale, Hülse“ in estn. kõba "Tannenrinde“, mordw. kuvo „Borke, Rinde, Kruste“, čer. $k u \beta o$ „Schale, Schuppe, Hülse, Schote“;

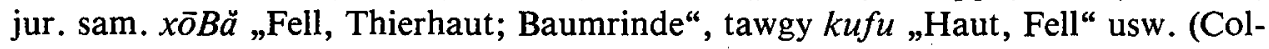
linder I 25; III 87):

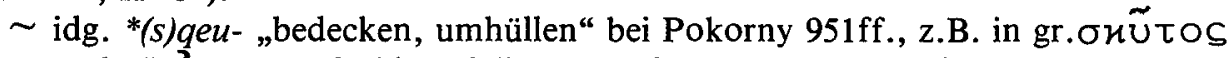
„Haut, Leder“, $\vec{\jmath}_{\gamma}-\mathcal{H} \cup \tau \ell$ „bis auf die Haut“, lat. cutis „Haut“, ahd. usw. $h \bar{u} t$ ds., schweiz. hut „Hülse, Fruchtschale“, mnd. schōde „Scheide“, F. „Schote, Erbse“, mhd. schōte „Schote, Samengehäuse“ usw.

Im Indogermanischen ist der urspr. nominale Stamm, wie auch sonst oft, zum verbalen Stamm geworden und die Bedeutung wurde stark erweitert, wie ja zu erwarten war, wenn die verbale Funktion mit sich auch eine Richtung ins Abstrakte herbeifuhr.

(7) unter ein gemeinsames ural. *kup- „Lunge; Fischblase“ kann man unterbringen:

a) ostj. kop-ək „the lungs“; sam. jur. kõp-uj, kap-uj, kap-y „Lunge“; urspr. *kup- (event. *kupp-) "Lunge“ + verschiedene Suffixe; s. Collinder, FUV. ${ }^{2}$ S. 45;

b) finn. kup-inas, ol. kup-ino, lapp. Inari gobpan, wog. qăa-en, xäp-en „fishsound" bei Collinder FUV. ${ }^{2}$ 108; s. noch SKES. s.v.;

c) finn. $k u p-s-u$ "fish-sound“, estn. kop-s „lung“, wog. $\stackrel{a}{p}-\check{s}$, qåp-s(i), xåp-si „Lunge“, qap-sej „Fischblase“ (Collinder ibd.); hier *kup + ein ś-Suffix; zu b) und c) vgl. auch SKES. 242 und 243:

$\sim$ idg. * ${ }^{*}$ wes- und *Kus- (seț- oder anit??) „keuchen, schnauben; seufzen“ (bei Pok. 631-632), a) "atmen, schnauben“ in ai. śvási-ti, them. - $a$-ti, davon av. suši „die beiden Lungen“; in der Bedeutung b) „seufzen“ ai. oben (auch a!) und „klagen“ lat. queror, toch. B kwəs-, präs. kwəs-nā-trə (von seț-Basis!) usw.; auch germ. Idg. a) ist von b) nicht zu trennen, trotz van Windekens, Le Tokharien I, S. 248; ural. kupsu (c) enthält dasselbe $s$-Element wie die idg. Wurzel. 
(8) ostseefin. *külpe- „baden, ein Bad nehmen“ in finn. kylpe- ds., kylpy „Bad“, kylvettä- „baden“, karel. olon. külbe-, lüd. külbe-dä- „baden, schwimmen“

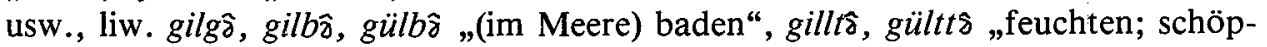
fen", "baden" bei Toivonen 254.

idg. *k'leu „spülen, rein machen“ (Pokorny 607) in gr. $\varkappa \lambda \circlearrowright \zeta \omega$ „spüle“, alat. cluō „purgo“, lat. clo(v)āca „Abzugskanal“, kymr. cli-r „hell, klar, heiter, rein“, got. usw. hlü-t-r-s „hell, rein, klar“, anord. hlér „See, Meer“ (<*kleuso-), lit. šlúoti mit dial. Präs. šlavù „fegen, wischen“ (urspr. „rein machen“), lett. slau-kšet „platschen, pladdern“ usw.

Es ist in diesem Falle mit einer urspr. dreisilbigen Wurzelform zu rechnen, die urspr. etwa *küle-pe- lautete; *-pe- wäre ein verbales $S x$, getreten an eine kürzere Wurzelform *küle-, für welche an beiden Seiten noch Beweise vorhanden sind, s. zum Idg. Pokorny a.a.O.; auch finn. kyly, karel.olon.lüd. külü „sauna“ bei Toivonen a.a.O.

(9) f.-ugr. *lepe- „Blatt“, sicher im Ugr.: ung. levél ds., (auch „Brief“), wog. lūptä, lapta ds., ostj. DN lïBət, Pl.liptət „Blatt“ usw., Vi. līwət „Blatt des Baumes, des Krautes“, Trj. !ı̣p $\hat{\partial} t$ „Blatt (des Baumes, Tabaks)“ usw., wozu noch finn. lepee-„,Wollflocke“ vielleicht gestellt werden kann (ugr. bei Szinnyei, Nyelvh.c 145; finn. Bárczi 190; vgl. auch Toivonen 288):

$\sim$ idg. *leu-bh-, *leu-p- ,abschälen, entrinden, abbrechen, beschädigen“ (Pokorny 690f. in lat. liber „Bast, Buch“ ( $<$ *lubhro-), alb. labë „Rinde, Kork“, air. luib "Kraut", got. laufs, lauf „Blatt, Laub“ = ahd. loub, d. Laube, lit. lubà „Brett“, lúobas „Baumrinde“, lett. luõbs „Schale“, russ. lub „Borke, Bast“; lit. lupù, lùpi „(ab)häuten, schälen“, russ. lupít „(ab)schälen“ usw.; die Bed. „rauben, Raub“ im Bsl. ist sicher sekundär, gr. lýp $\bar{e}$ „Kummer, Trauer, Schmerz“ bleibt wohl fern (vgl. Frisk, Gr. EW. II 145f., der auch ai. lumpáti „zerbricht“, beschädigt, plündert" besser beurteilt als Pokorny a.a.O.); so kann man die urspr. Bedeutung dieser Wurzel als „abschälen, entrinden“, weiter „abblättern“ (vgl. lett. laupît) festsetzen, alles demnach vom Abstreifen der Pflanzen bzw. Pflanzenteile (Stamm, Ast). Die Konsonanten *-bh- und - $p$ - sind natürlich rein idg. formantische Elemente. Am ehestens ist es mit einem sog. privativen Verb auszukommen, wie d. köpfen; also „Blätter, Rinde, Schale usw. entfernen“?

(10) f.-ugr. *repe- „zerreissen, spalten“ in ung. reped „einen Riss oder Sprung bekommen, aufspringen, platzen, reissen“, repeszt „sprengen, spalten“ usw., finn. repe- $\ddot{a}$ „einen Riss bekommen, sich spalten, bersten, reissen, zerreissen..." (Inf. revetä), repi $=$,zerreissen (trans.), reissen u.a.)" (Prät. 1. Sg. revi-n) (Szinnyei, Nyelvh. ${ }^{7}$ 39; Bárczi 256; Toivonen $768 f$., wo auch lapp. U, Pi., L rahpat, $\mathrm{N}$ râppât „avata, öffnen, aufmachen, aufschlagen“ zitiert wird):

$\sim$ idg. *reu- usw. „,aufreissen, graben, aufwühlen; ausreissen; raffen“ (Pokorny 868) in ai. rav- ,zerschlagen, zerschmettern“, gr. $\stackrel{\varepsilon}{\rho} \rho \sigma i-x \vartheta \omega \nu$,die Erde aufwühlend“, lat. $r u \bar{o}$ „aufreissen, wühlen, scharren“, anord. $r \bar{y} j a$ „den Schafen die Wolle ausreissen“, lit. ráuti „ausreissen, ausjäten“, aksl. ryti „graben“; a.O. $869 \mathrm{ff}$. 
zahlreiche Ableitungen, darunder *reu-p- „ausreissen, zerreissen, brechen“ mit lat. rumpō usw.

Die Ableitungen auf idg. $-b-,-d-,-d h-,-q-,-p-,-s-$ machen wahrscheinlich, dass die Wurzel seit je mit $r$-angelautet hat und dass sie nichts mit idg. *ereu- ,aufreissen" bei Pokorny 338 gemein hat; das letztgenannte ist wohl in ein *er- „aufreissen“ + nominale Suffixe ${ }^{*} u s-$, ${ }^{*}$ no-, *-ues- aufzulösen.

Toivonen 769 vermutet deskriptiven Ursprung für die finn.-lapp. Sippe, was mutatis mutandis - auch für die idg. Wurzel vermutet werden kann; doch scheint ein gemeinsamer Ursprung aus indoural. (onomatop.?) *repe- ratsam zu sein.

(11) Toivonen, FUF. XV (1915) 68 verbindet ung. nyár-fa „Pappel, Espe“ mit sam. jur. núrr-ka „Espe"; während im Sam. ein $k k$-Suffix vorliegt, ist das ung. Wort eine Zusammensetzung mit fa „Baum; Holz“, das bekanntlich ein uraltes Wort ist, ural. *pū „Baum" in finn. puu ds., „Holz, Brennholz“, tscher. pu „Holz, Brennholz“, wotj. -pu „Baum; Holz“, syrj. pu, wog. -på „Baum“, ung. fa; jur. sam. $p \bar{a}$ usw. „Baum, Holz, Stock, Klotz" usw. (s. Collinder I 53; usw.); dies Wort kommt auch sonst als zweites Glied der Zusammensetzungen vor, die Baumarten bezeichnen: vgl. wotj. kǐz-pu mit Vorderglied $\sim$ finn. kaski ,junge Birke", syrj. kić-pu „Birke“" neben kij ds. (vgl. Collinder I 86); wotj. susi-pu „Juniperus communis“, syrj. sus-pu „Pinus cembra“ (Collinder I 58); usw. Man kann solche Zusammensetzungen auch fürs Idg. vermuten:

$\sim$ idg. $u$-Element in einigen Baumnamen usw.:

a) idg. *dere-u- „Baum“ bei Pokorny $214 \mathrm{ff} .$, z.B. in ai. dāru „Holz", av. dāuru

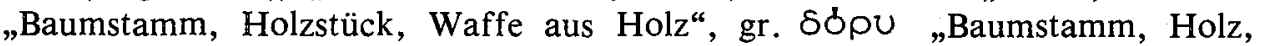
Speer", maked. $\delta \& \rho u-\lambda \lambda$ oS „Eiche“, alb. dru "Holz, Baum, Stange“, dru-shk „Eiche“, kymr. derw-en „Eiche", got. usw. triu „Holz, Baum“, aksl. drěvo „Baum“, heth. taru „Baum, Holz“ usw.; wenn nun dies Wort eig. eine Zusammensetzung mit unserem Wort für „Baum, Holz“, ural. * $p \bar{u}$, ist, so verbinde ich das Vorderstück ( = einst 1. Glied der Zusammensetzung) mit. ural. *ńerke „Spross usw." bei Collinder I 43. a. in ung. nyir (Stamm nyire-) „Spross, Ried, Stengel; Zweig; Birke“, čer. nöryö „Spross, Zweig; junger Baum“, wotj. noör „Spross, Gerte, Zweig“, wog. ńir, ńər „Ried, Gerte, Zweig“, ostj. ńor „junger Wald, Dickicht (auf alten Brandflächen), mit jungem Wald bewachsene alte Brandfläche" usw.; sam. jur. ńérū „Weide“ usw.; im Idg. urspr. *dereH-u- „Zweig-Holz o. dgl.“, mit Verlust des Laryngals unmittelbar vor ${ }^{*}-\underline{u}$ - (mehr an einer anderen Stelle); dass Idg. einst sicher auch einen $u$-losen Stamm ${ }^{*} \operatorname{dere}(H)$ - $=$ ural. *nerke kannte, beweist gr.

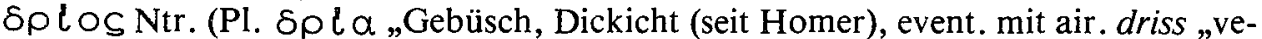
pres" ( $<* d r i$-st-), nach Frisk, Gr. EW. I 418 wohl zu *dereu-, obwohl morphologisch ganz unklar; jetzt kann man ein neben *dereu-, besser ${ }^{*}$ dereH-u-, stehendes *dere- $i$ - (mit $i$-Suffix) ansetzen; zu ural. $\dot{n}$ - $=\mathrm{idg} . d$ - weitere Beispiele in meinen Indouralica VI;

b) gr. $\pi \tau \varepsilon \lambda \varepsilon \alpha$, epid. $\pi \varepsilon \lambda \varepsilon \alpha$ „Ulme, Rüster“ = myken. pte-re-wa, lat. tilia "Linde“ und wohl noch ahd. fel(a)wa „Weide“, osset. färue „Erle“ (vgl. Boisacq, 
DEGr. 820; Pokorny 847 mit falsch angesetzter Urform; Frisk, Gr. EW. II $611 \mathrm{mit}$ anderer Deutung des gr. Wortes); zur ural. Verwandtschaft dieses Baumnamens vgl. Čop, Orbis XIX (1970) 299: zu finn.-ugr. *pić( $\left.a^{x}\right) l / r a$ - „Sperberbaum, Eberesche“ in finn. pihlaja, pihlava usw., wozu Toivonen, FUF. XIX (1928), 201f., Nr. 387, und Collinder I 107.

Idg. *dereH-u- „Zweig-Holz“ ist ganz mit ung. Kompositum nyir-fa „Birke, Birkenbaum“ und tscher. nör-pö usw. „Alnus“ identisch; und im Fall b) kommt zu idg. *ptele-u- (daneben tilia eventuell aus *ptele-i- wie $\delta \rho l O S$ im Fall a) neben uStamm) als Rest einstigen Kompositums im Finn.-Ugr. ebenfalls entsprechende Zusammensetzung mit *p $\bar{u}$ „Baum; Holz“ in wotj. paleź-pu usw. „Vogelbeerbaum, Spierlingsbaum"! Natürlich wäre es tollkühn, die eben angeführten finn.-ugr. Komposita als so alt zu nehmen, dass auch sie genetisch mit den uridg. Baumnamen auf -w-total identisch wären. Da andrerseits das Idg., das alte eurasische Wort für „Baum, Holz“ im selbständigen Gebrauch wohl nicht mehr besitzt, müssen die Wörter *dere-H-u und *p(t)ele-w- recht alte Komposita oben ausgelegter Art sein.

Es folgt eine Etymologie, die bedeutungsmässig sehr gut gewählt wäre, jedoch wegen der phonetischen Struktur im Lapp. und Mordw. gewisse Schwierigkeiten mit sich bringt (einzige Möglichkeit: das idg. *-eu/u- ist sekundär altem (iu:) monophthongischem, aber echt höchstem Vokal der hinteren Reihe hinzugebildet worden?

(12) nach SKES.625 bedeutet finn. puhde „Zeit des Wachens vor Tagesanbruch, Frühstunde"; dazu gehört das Verbum *pukta-, erhaiten in lapp. boktet "wecken“, weiter mordv. puv-ta-ms, puf-ta-ms „erwecken, aufwecken“. S. noch Toivonen, FUF. XIX (1928), S. 113 Nr. 143; übrigens noch Posti, FUF. XXXI (1953), SS. 46-48 (s. unten):

idg. *bheudh- „wach sein; wecken, beobachten; geweckt, geistig rege sein usw." in ai. bö́dhati/-tē „erwacht, erweckt, ist wach, merkt u.a.“, gr. peúthomai und pynthánomai „erfahre, nehme wahr“, lit. bundì „erwache“, baudinti „aufmundern“, got. ana-biudan „befehlen, anorden“ und viele andere; s. bei Pokorny 150 -152. Die Urbedeutung „erwachen; anbrechender Tag“ ist auf beiden Seiten gleich, nur die Phonetik macht etliche Schwierigkeiten: nach Posti a.a.O. sollte in den westfinn.-ugr. Sprachen wenigstens in éinem Teil der $k t$-Belege diese Gruppe mit reinem $-k$ - erhalten und die $p t$-Wörter von den ersteren treu auseinanderhalten bleiben: meist siegt $-k t$-, teils $-v t-,-f t$ - (so mordw.!). In unserem Fall ist also die Urform unsicher und in $-h$ - nur möglicherweise Existenz bzw. Resultat eines einstigen $-p-=$ idg. $-u$ - des Wurzeldiphthonges zu suchen.

(13) im Finn. (und Mordv.) gibt es eine Wurzel (s. SKES. 1158)*(t)säáke- „funkeln", die im Idg. als *saH- erscheinen müsste; finn. auch säen, Gen. säkenen, "Funke" mit mordw. séjern, usw. „Feuerschwamm" (mit Suffix -ma/mä). Nun hat diese f.-ugr. Wurzel tatsächlich Vertreter im Idg., denn finn. dial. existiert ein (altes) Partizipium auf $*_{-}$pa/pä (worüber noch unten bei Wortbildungslehre) $=$finn. säkevä „stark funkelnd“; mutatis mutandis erwartet man auf indogermanischer Sei- 
te ein *sáHw-, das man in der idg. Bezeichnung der "Sonne", bei Pokorny, Idg. EW. 881ff. unter *sāwel, *sül-, wiederfindet; so meine Mitteilung vor den Linguisten in Regensburg 1979. Détails und Polemik an anderer Stelle; zur Flexion auch Cop, Dekl. 90-91 usw.

Die idg. Wurzel *sáHw- ist also eine willkürliche Abstraktion aus einem fertigen Wort (Partizipium auf unser Suffix - $p a$ ). Die Varianten in verschiedenen idg. Sprachen, *sá̀wel; *sHuwél-, * ${ }^{\rtimes} \mathrm{Hul}$-/sHul-, schliesslich *suHul- (mit Assimilation nach Kurylowicz 1927) bilden eine ganz regelmässige Abwandlung einer ursprünglich zweisilbigen ( ${ }^{*} \mathrm{saHe}-\mathrm{w}$-) bzw. sogar dreisilbigen Basis (*saHe-we-).

(14) finn.-ugr. *ș̆upa „nicht dauerhaft, schwindend“ in finn. hupa „von kurzer Dauer, bald vergehend; unzulänglich, ungenügend; verschwenderisch; Zeitvertreib; schwach, schlecht", estn. huba usw. "mürbe, spröde, zerbrechlich, locker; usw.", vgl. auch finn. hupaan mene- „magrer werden, abzehren", mordw. čova (E), ̌̌s sa

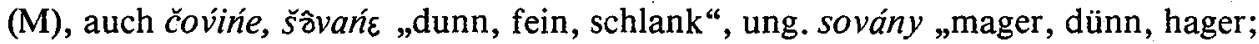
armselig, dürftig, kärglich“ (Bárczi 273; Szinnyei, Nyelvh. ${ }^{7} 39$, 95; Collinder I 82; III 52, 87; Toivonen 89f. usw.):

$\sim$ idg. *suendh- „schwinden“ (mit ? bei Pokorny 1047) in ahd. swintan „schwinden, abmagern, welken, bewusstlos werden“, asächs. far-swindan „verschwinden“, ags. swindan „abnehmen, schwinden“, Kaus. mhd. swenden „schwinden machen, ausreuten" u.a. unsichere Wörter (sl. u-vędati "welken" gehört wohl mit voditi, qditi "räuchen“ zu einem ganz verschiedenen idg. *uendh- „brennen“); weiter hierher idg. *su- $\bar{l}$ - usw. in anord. svia „nachlassen“, svina, ahd. swinan „abnehmen, schwinden" u.a. bei Pokorny 1052.

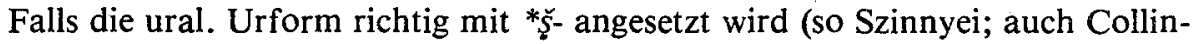
der III 411, versehentlich? - vgl. dagegen ibd. 52, wo ${ }^{*} \breve{c}$ - angesetzt wird), ist der idg. Verbalstamm *suendh-mit intrans. $-d h$ - aus der $n$-Ableitung (Adjektiv) hergeleitet worden, die auch in ung. sovány und mordw. E čoviné usw. vorliegt; idg. suiist eig. *sue-i mit denominativem $i$-Suffix, abgeleitet direkt aus dem Adjektiv *sue$=$ ural. *ṣ̆upa; zum Vokalismus und zum Typus vgl. unten Nr. (29) *kpe-i- ural. *kota.

Wenn aber das ural. Wort ein $\breve{c}$-Wort ist, so ist die obige Zusammenstellung zu tilgen und durch die folgende zu ersetzen: idg. *dheu- „hinschwinden, bewusstlos werden, sterben“ in got. diwans „sterblich“, ahd. touwen, asächs. dōian, anord. deyja „sterben“, got. usw. daups „tot" und daupus „Tod“, ahd. tawalōn "hinschwinden, hinsterben" usw.; diese Wurzel ist als Verbalisierung des eventuellen indoural. *Dupa ( = ural. *çupa, wenn so anzusetzen) zu nehmen; rein denominativer Verbalstamm mit Suffix * $i$ - (und wohl auch einem weiteren laryngalen Element davor) ist aber in *dhue $i$-, *dhui zu finden: arm. di „Leiche, Leichnam“, air. dith „Ende, Tod“ (beide aus $d h u_{n}-\bar{l}_{-}^{-}(-)$, ags. dwinan „abnehmen, schwinden“, anord. $d \bar{l} n a$, divena ds, u.a. 
Welche der vorgebrachten Verknüpfungen richtig ist, wird wohl die Zukunft zeigen, denn ausserhalb des Indouralischen gibt es noch weitere mögliche Verwandte, die im obigen Dilemma entscheiden können.

Anm.: Damals (1973) war ich überzeugt, dass alle drei ural. dentalen Frikativen ( $s, s$ und $s$ ) gleichermassen im Indogermanischen das Archiphonem /s/ ergeben; aus dem numerischen Verhältnis des /s/ zu ural. drei Sibilanten ergibt sich, dass die Frequenz des idg. $s$-Lautes eigentlich Summe der drei Frequenzen der ural. obgenannten Sibilanten sein muss; dies gilt natürlich nur für den Fall, dass keine der obigen Frequenzen durch anderweitige reichere Quelle stark vergrössert wurde, was durch das Material gerade des vorliegenden Aufsatzes geschehen ist: die Frequenz des idg. einzigen $s$-Lautes sollte demnach um ein Drittel grösser sein als die Frequenz der ural. Sibilanten zusammen. Da darüber hinaus

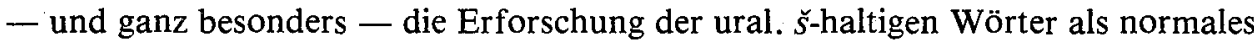
Gegenstück des ural. / $\breve{s} /$ indogermanisches $/ \mathrm{h} /$ darbot, musste diese letztgennante Lösung des Problems akzeptiert werden und die neue Auffassung im Aufsatz XIX der IU.-Reihe Platz finden. Die Veröffentlichung des Materials ging aus verschiedenen Gründen sehr langsam vor sich, bis heute nur stückweise als gelegentliche Unterlage vereinzelten Etymologien, so z.B. im Aufsatz IU. XVII, wo gezeigt wird, wie das dem ural / $\breve{s} /$ entsprechende idg. /h/ grosse Rolle bei der Entwicklung des idg. Vokalismus gespielt haben soll.

$\mathrm{Zu}$ bemerken ist - so schon hier oben - dass manche Forscher vor der Rekonstruktion des ural. Urlautes sowohl bei mutmasslichem $\check{s}$-Laut wie bei seinem Konkurrenten (der Affrikata $\breve{c}$ bzw. ț̆ $)$ schwanken; oben im Texte ist darauf schon hingewiesen worden. Immerhin hat MNyTESz. III s.v. sovány mit einem Ansatz *çupa wohl Recht. Zu einem weiteren Päne-Synonym vgl. meinen Aufsatz Coll. Ieur. I (Ljubljana 1978), SS. 163f. (ung. dög „cadaver“ u.a.).

Unser sovány gehört samt Sippe in einen anderen Kontext, am ehesten in die Gruppe der ural. echten $\breve{c}$-Worter, worüber weiteres in IU. XIII.

Um auf die Frage des $\breve{s}$-Lautes im Ural. und seiner Entsprechung $h$ im Idg. zurückzukehren, erwähne ich nur soviel, dass einige solche in der Studie vom Verfasser dieser Zeilen „Méditerranéen et Indo-Ouralien“ (in Lingu. XVI/II (Ljubljana 1976), passim, zur Sprache kommen: SS. 13f. (*lešmä „Kuh“), 18-19 (*šäntä „Getreideart, Gerste“), 20 (*šejte „Wald, Gestrüpp“), 22-24 (*p/bäšä „Nuss; bákkar"); s. noch darin: Conclusions pt.

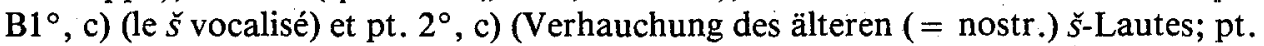
D, S. 31 Mitte. Weitere IU.-Gleichungen in betreff des einstigen $\breve{s}$-Lautes kommen u.a. in folgendem vor:

a) in Lingu. XIV (Ljubljana 1974), S. 41 (fin. hepo, hevonen "Pferd“ zu idg. *ékwos, iu. *ekș̌epa-);

b) in meinen Vorträgen aus dem Gebiete der indogermanischen Pho- 
netik habe ich hie und da auch die indogermanischen Reflexe der iu. $\breve{s}$ Laute berührt, so in Regensburg 1979, wo ich meine Ansichten über das frühindogermanische Laryngalsystem dargelegt hatte: ich stellte fest, dass die. Entwicklung der indogermanischen Gutturale (der $k$-Laute intervokalisch, der $\gamma$-Laute frei) zu einem Ergebnis führte, wo es drei $x$-Laute und drei $\gamma$-Laute gab, also drei Paare der Gutturale, differenziert nach der Artikulationssteile, wie bekanntlich auch sonst auf dem Gebiete der idg. Gutturale; dazu kam noch ein $h$-Laut (Hauchlaut), der dem uralischen $\check{s}$-Laut entsprechen sollte; also:

\begin{tabular}{|c|c|c|}
\hline & stimmlos & stimmhaft \\
\hline $\begin{array}{l}\text { velar } \\
\text { palatal } \\
\text { labiovelar }\end{array}$ & $\begin{array}{l}-x- \\
-x^{\prime}-=-x^{\prime}- \\
-x^{u} u\end{array}$ & $\begin{array}{l}-\gamma_{-} \\
-\gamma_{-}^{\prime} \\
-\gamma u_{-}\end{array}$ \\
\hline Hauchlaut & $\ddot{-h}$ & $(-\gamma-)$ \\
\hline \multicolumn{3}{|c|}{ Weiteres in IU. XVII und XIX. } \\
\hline
\end{tabular}

(15) ugr. *tipe- od. *täpe- „sich verirren“ in ung. téved „(sich) irren, im Irrtum sein; sich verirren, irregehen, auf Abwege geraten“, wog. top-. tip-, ostj. DN $t \partial p$-,sich verirren, sich verlaufen“, Vj. $t \partial w$ - usw. „sich verirren“, vgl. Trj. təpəytə-,,verirrt sein, verirrt umherwandern“ usw. (Szinnyei, Nyelvh. ${ }^{7}$ 154; Bárczi 309 u.a.):

idg. *tew „umherwandern; zögern, zaudern" (so ist die Gruppe unter *tūlo- „säumig und langweilig im Arbeiten, Reden usw.“ bei Pokorny 1098 zu verbessern), u.a. in: slvn. távati „herumtappen; unsicher gehen“, o-távljati „verzögern, verschieben“, ob-o-távljati se „zaudern, zögern“, lett. taũjât „unentschlossen, im Zweifel sein, nicht wissen, was man wählen soll, tasten“, lett. taũnâa „zögern, zaudern, nicht fertig werden, langsam sein“, $t \bar{u} l$ 'ât „säumen usw.“, slvn. noch $z a-s$ túnj/ónj „umsonst“ (sl. *tunée), lit. taujóti „umherwandern“, s. C̆op, Slav. Rev. XIII (Ljubljana 1961-62), SS. 197ff.; das germ. Material bei Pokorny a.a.O.; Jóhannesson, Isl. EW. $450 f$.

(16) in SKES. 100 findet man ein finn.-lapp. Wort, das auf eine Urform *šäpä „Schopf, vorspringender Knochen zwischen Hals und Rücken eines Pferdes“ (auch einer Kuh, eines Ochsen) zurückgeht; daneben Bedeutungen „Mähne“ usw.; die Sippe liefert auch eine Variante mit $s$-Suffix: also finn. hävä-s, Gen. häpään $(<$ *šäpäse-n); lapp. N sâeppe, R sepe-s usw.:

idg. Sippe bei Pokorny, IEW. 87: *aug'h-, *uǵh- „Genick in ai. uṣ̆ṇíhā ds., gr. aukhén „Nacken“ u. viele andere, s. auch Pokorny 1173 unter *wes- „drehen, wickeln“ (dies abzulehnen). Dazu stelle ich weiter sl. vyja (russ. výja, aksl. vyja „trákhēlos, aukhến“ (vgl. Vasmer, Russ. EW. I 243: ohne Etymologie, m.E. aus 
einsilbigem idg. ${ }^{*} \bar{u}$-s, Gen. ${ }^{*} u w$-és) und arm. viz „collo; cervice" nach CIAKCIAK, m.E. ganz einfach aus idg. *us-iǵhä; Einzelheiten anderswo, hier nur soviel noch, dass die idg. Sippe in der Lautstruktur gemäss unserer iur. Lautentsprechungsregel $w=$ ural. $-p$ - enthält und dass sie in zwei Varianten - in betreff der Wortbildung erscheint: in éiner ohne Suffix $*_{-s-}$, in der anderen aber mit diesem Bildungselement; anl. *̌s- der ural. Wörter $=$ idg. ${ }^{*} h$ - bzw. Ø-. Trotz der stark veränderten Lautung also totale Identität.

(17) das f.-ugr. kipinä (mit zahlreichen Varianten, s. SKES. 197) „Funke“, verbal kipinöid̈̈ „funkeln, sprühen“ gehört nach SKES. zu syrj. kiń „Funke“, weiter zur Gruppe mit kyv/kyp- in lüd. küben, estn. kübe usw. (bedeutungsmässig und formal ist säkene „Funke" nahe verwandt), auch lapp. $\mathrm{H}$ gâppâ, $\mathrm{R}$ kappanjes "Asche“:

$\sim$ idg. bunte Gruppe, die aus einem Urwort *Keu- (= f.-ugr. *kipe-) „leuchten" hervorgegangen ist: a) Urwurzel *keu- ds. (Pokorny 594-595, u.a. in av. *savah- „Morgen, Osten“ (dazu savah-i- „Name im Osten gelegenen Erdteils“), ai. śvás „cras" u.a., ai. śó-na- "rot" und nahe verwandte $n$-Bildungen, b) Wurzelerweiterungen mit - $b h$ - und - $d h$ - (ai. súundha-, śúmbha- usw.), c) in Erweiterungen *kwei$t / d$ - u.a. (sl. světiti usw., d) in der Wurzelform ${ }^{*} k w$-en- in *kwénto-s „heilig “ (av. spanta-, lit. šveñtas, sl. svętర̈ mit av. span-ah- „Heiligkeit“ u.a. bei Pokorny 630); vgl. noch Pokorny 628-629; das in d) stehende $n$-Element kann mit demjenigen der f.-ugr. Gruppe historisch identisch sein.

(18) f.-ugr. *upeš „stallion, bull, ram" steckt wahrscheinlich in finn. uve, Gen. upeen ds., vgl. lüd. ubeh usw., vgl. SKES. 1569; Posti, FUF XXXI (1953-1954), S. 4; formell, sehr wenig aber bedeutungsgeschichtlich (wozu ebenso misslich čer. òžz und perm. $u z \bar{z})$ :

$\sim$ idg. *owi-S „Schaf“ (zu „ram“ oben etwas näher) (Pokorny 784) in ai. ávi-, lat. ovis, lit. avis, anatol. hawi-š usw.; idg. ${ }^{*} H o$ - hat regelrechtes -o- (aus der Folge $-u C e$-) und $* H$ - aus älterem $\breve{s}$-Laut durch Antizipation aus dem Stammauslaut (ähnlich wie im Griechischen sehr oft, vgl. i $\varepsilon \rho \delta \varsigma<*$ ih-). Das $-h=$ ural. $-\check{s}$ kann sogar in got. Kollektiv awe- $\theta i$ stecken (d.h. $\left.={ }^{*}(h) o w e ́ h-\right)$.

(19) ein ural. *korpe- „brennen, flammen, lodern“ steckt in finn. korventa „brennen, sengen", auch korveta $\sim$ korpea-, lapp. $\mathbf{N}$ gourbâdit und Lule guorbâ-, „get damaged by forest fire“; selk. küram, kürram „sengen “ (vgl. Collinder, FUV. ${ }^{2}$ 45; SKES. 221-222 usw.):

ai. krúdh-ya-ti „gerät in Zorn", krödha- M. "Zorn" usw., s. Mayrhofer, Kzgef. EW. I, S. 280, ohne Etymologie. Da „Zorn" oft mit innerem Feuer verglichen wird (vgl: lat. ardeo und $\bar{\imath} r \bar{a}$ incēnsus), kann die ural. Anwendung die ursprüngliche sein. Ural. *korpe-, *korpe-nta- usw. sind regelrechte Bildungen, z.B. -nt- Frequentativum (dazu vgl. Čop, Orbis XXII (1973), SS. 5-42, bes. SS. 26-27, zum Vokalismus (idg. vorablautlich *korpe-nt-) das Paar finn. korento: idg. *ghrendh- S. 24!). 
(20) lapp. njarbâd „thin (of fluids or semifluids); thin, sparse (of forest, grass, hair, shoal of fish); short-haired, sparsely wooded“; sam. jur. nierwed „thinly netted" bei Collinder, FUV. ${ }^{2} 58$ usw.:

idg. *(s)ner-wo-, *(s)nor-wo- bei Pokorny 975-977, bes. 976, u.a. in asächs. naru, ags. nearu „eng“ (urspr. „zusammengeschnürt“), ahd. narwa F., narwo $M$. „Narbe“ usw.; m.E. auf einem *ner-w-eto- beruht arm. nergev ,tenuis, gracilis“. Die idg. Gruppe ist angeblich deverbal, was von lapp. Worte nicht zu beweisen ist. Bedeutungen wie bei tenuis u.a.

\section{Regel}

Uralischem intervokalischem schwachem -t- entspricht auf der indogermanischen Seite der reine Sibilant $-s-$; die Struktur der Urstämme (= Wurzeln) ist entweder CVtV oder (seltener) VtV im Uralischen, während im Indogermanischen der zweite Vokal normal fehlt (Vokalreduktion bzw. isomorphische Analogie nach falscher Analyse, s. oben unter Kapitel über - $p$ - (zur Ursache dieses Umstandes vgl. C̆op, IU. XVII und XVIII - in Druck bzw. in Vorbereitung - auch Dekl. 86-105 passim; Lingu. XIV (1974), SS. 41-53; u.a.). Die indogermanischen Entsprechungen sind demnach um éinen Vokal kürzer, die ehemalige intervokalische Lage somit manchmal nur Postulat der idg. Akzent-bzw. Ablautlehre.

(21) f.-ugr. *itä- „sich erheben, aufsteigen“ und nominal „Erhebung, Aufstieg“ in finn. it $\ddot{a}$ - „keimen, sprossen“, itä „Osten“, itu „Keim, Spross“, lapp. itte$i \delta e-$ „erscheinen, sich zeigen; zu spriessen anfangen (Gras. usw.)“, itta „zum Morgen

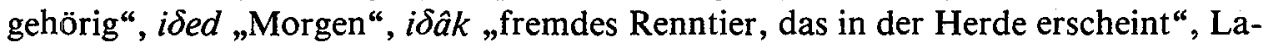
gercrantz ihtie- „erscheinen, sichtbar werden, auftauchen, zum Vorschein kommen, sich zeigen“, auch „aufgehien (Sonne)“, ostj. et - „sich erheben, hervorkommen, herausspringen, wachsen“ (Gras, Pflanzen, Blätter), V auch „sichtbar werden (Mond)“, Trj. àt- „sich erheben (Gras), sichtbar werden (Blatt, Mond); eintreten, geschehen“, Ni. et- „aufstehen, zum Vorschein kommen (hinter etw. hervor); aufgehen (Sonne); sich auf den Weg machen; glücken, gut ausfallen (Fang, Arbeit) " (Collinder I 83; III 81; Toivonen 111, der zweifelnd noch wotj. $u d$ - „hervorwachsen (Pflanzen aus dem Boden)“, udan „Spross, Sprössling“, heranzieht und überhaupt eine neue Gruppierung erwägt):

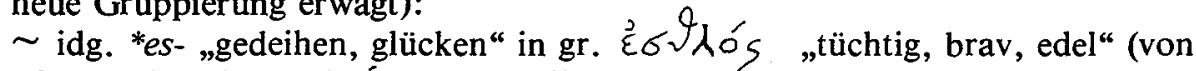
Menschen und Sachen), ai. é dhatēe „gedeiht", av. azdya- "wohlgenährt", vgl. Mayrhofer, Aind. EW. I 128 (und 560); Frisk, Gr. EW. I 574; Pokorny 342 (alle mit Lit.); alles scheint darauf hinzuweisen, dass in idg. *'z-dh-etoi ein intransitives $d h$ Präsens vorliegt (vgl. Brugmann, Grdr. ${ }^{2}$ II 3/I, 374), das urspr. vom Pflanzenwuchs gebraucht wurde, eine Bedeutung, die im Ural. im Vordergrund steht.

(22) Die urspr. Bedeutungsnüance, "sich erheben" und (beim nominalen Gebrauch der Wurzel) „Erhebung, Aufstieg“ kommt andrerseits im folgenden idg. Adverb vor:

a) heth. ̌̌arā „empor, hinauf, herauf“, als Postposition „auf etwas hinauf“, luw. šarra "sur" (Präposition); 
b) heth. šer „oben, oberhalb, darauf; darüber; dabei, dafür, deswegen“, als Postpos. „auf, oben in; für, wegen, betreffs", luw. šarri „en haut, sur" (Adverb und Präposition), lyk. hri „auf“; eine Zusammensetzung ist lyk. hr-ppi „pour, sur“.

Zum Ganzen Laroche, BSL. LIII (1957-58), 178ff., zur Morphologie Čop, Lingu. VII (Ljubljana 1965), 103. Wir haben demnach mit einem alten Lokativ *sér-i und einem Lativ *ser- $\dot{a}^{x}$ (vielleicht im letzteren ganz im Sinne Laroches eher reduktionsstufig * $e_{r-a ́ x} x$, doch postuliert das luw. Wort šarra unbedingt vollstufiges *ser-, s. Verf., IF. 75 (1970), S. 86) zu tun. Nun ist séri (woneben ein endungsloser Lok. sér zugegeben werden kann) genau so gebildet wie *up-ér, *up-éri „über, oberhalb“ bei Pokorny 1105; dies ist seinerseits zum Verbum heth. up-zi "geht auf" (von der Sonne usw.) zu stellen (zum letzteren Pokorny 1107), das auf idf. *eup-ti "erhebt sich, geht auf" fusst (s. Verf., Lingu. XI (Ljubljana 1971), 42f.). Da nun in *up-ér-i, *up-ér wie auch sonst in Adverbien auf *-ér(i) der Akzent auf dem $r$-Suffix steht, die Wurzel (Verbalstamm?) aber in Tiefstufe steht, ist dasselbe auch für unser *sér, *séri zu vermuten; die Lativform auf ${ }^{*}-a x$ ist wohl eine erst anatolische Hinzubildung zum alten Lokativ. Auf diesem Weg kommen wir zur Analyse in die (tiefstufige!) Wurzel * $s^{-}+$Suffix *ér(i); und an der Hand der historischen Bedeutungen muss diese Wurzel etwas wie "Obiges, Erhöhtes" bedeutet haben; wir gehen wohl nicht fehl, wenn wir neben diesem Adverb *s-ér(i) nun noch ein Verbum *ás-ti „erhebt sich, geht auf, nach oben" ansetzen (genau so wie *éup-ti: *up-ér(i)!); dies Verbum ist bis auf die kleinste Kleinigkeit mit finn.-ugr. *itä- „,ich erheben, aufsteigen“ identisch; nur muss man a) die Entsprechung ural. $-t-=$ idg. $-s$ - und b) die Wirkung der idg. Vokalreduktion (der zweite Vokal fällt im Verbum weg) in Betracht ziehen.

(23) ural. gibt es einen Verwandtschaftsnamen, der auch im Indogermanischen zu erscheinen scheint: finn. nato "the sister of the husband or the wife; the wife of the brother"; auch "sister-in-law"; čer. nuסo "the younger sister of the husband..."; sam. jur. $n \bar{a} d o$ "the younger brother of the wife...", $n \bar{e}-n \bar{a} d o$ "sister in law" (= „Schwägerin“) (Collinder, FUV. ${ }^{2}$ 56):

idg. *snusó-s „Schwiegertochter“ (Pokorny 978) in arm. nu, Gen. nuoy, gr. vuós, lat. nurus, sonst sekundär zu $\bar{a}$-Stamm umgebildet wie ai. snuṣ̆ă, akṣl. snzxa, ahd. snur, snora.

Bedeutungsmässig ist die Gleichung verständlich, da auf beiden Gebieten spezielle gesellschaftliche Normen obwalteten. Phonetische Schwierigkeiten sind nicht unüberbrückbar: ural. $=$ urspr. ${ }^{*}$ nata- $w$, im Idg. aus urspr. *nasá-w- über *nséuzu *snu- usw.

(24) ural. *jüte „Nacht, Abend“ in tscher. jüt „Nacht “, wog. jēt', iit' „Abend,

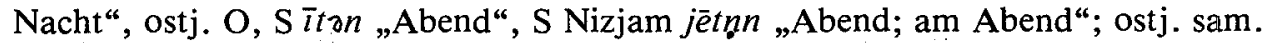
$\bar{u} t e, \bar{u} d e$ '„Abend“, kamass. nōd'i ds. nōdin „am Abend, koibal. nuide „Abend“ (Collinder 20):

$\sim$ idg. *g'( $\delta) h i e ́ s$ u. ähnl. „gestern“ (s. Pokorny 416) in ai. hyás ds. av. zyō, gr. el. $\sigma \varepsilon \rho \delta \varsigma$ : $\times \vartheta \hat{\varepsilon} \varsigma$ Hesych., sonst. $\times \vartheta \varepsilon \varsigma$, , alb. dje ds., auch "morgen“, lat. heri, 
hes-ternus „gestrig“, air. in-dé, kymr. doe „gestern“ (brit. aus *gdilies), got. gistradagis „morgen“, ags. geostra „gestern“, ahd. gesterōn usw. ds., anord. $\bar{i}$ gäer „gestern" $\left(<{ }^{*} g^{\prime} h \bar{e} s\right)$.

Man suchte allerlei Erklärungen für das idg. Wort, die aber ohne anderweitiges Vergleichsmaterial alle nur glottogonische Spekulationen blieben; ausführlich referiert darüber Walde-Hofmann, LEW. ${ }^{3}$ I 643; schon der Versuch, darin das idg. Wort für „Tag“ (Wurzel *dei-) zu sehen, muss bedenklich stimmen, denn die Wörter für "gestern“ fussen gewöhnlich auf dem Begriff „Abend, Nacht“, vgl. slvn. včera(j) „gestern“ zu večer „Abend“, lit. vãkar „gestern“ zu vãkaras usw., slvn. noch sinoči „gestern abend" von 56 „dieser“ + noč „Nacht“. Sich auf dies letztere si-noči berufend kann man auch im idg. Wort für "gestern" eine einstige Benennung eines bestimmten Abends: „des gestrigen Abends" sehen; wie schon vermutet, ist in *gh- der idg. demonstrative Pronominalstamm *ghe- usw. wiederzufinden (zum letzteren Pokorny 417f.), der Rest unseres Wortes ist aber die Entsprechung des ural. Wortes für „Abend, Nacht“, urspr. Bedeutung des Ganzen demnach „an diesem Abend, in dieser Nacht" wie in slvn. si-noči; *g'hiés, *g'dhés, *g'shiiés ist demnach endungsloser Lokativ mit der idg. Entsprechung von ural. *jüte als zweitem Glied, wo jedenfalls das $*_{-s}=$ ural. $*_{-t}$ - regelrecht ist. Alles andere, vor allem der Wechsel $-\delta-\sim-i$, muss einer späteren Untersuchung vorgespart werden; s. Indouralica VI.

(25) ural. *kato- (verbal) "weiden" und *kato (nominal) "Weide" in lapp.

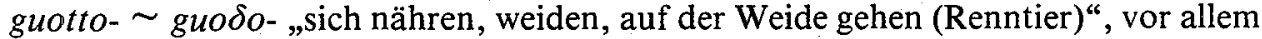
im Winter, Lule guotto „Viehweide, Weideland“; jur. sam. $x a \delta \bar{u}$ „Winterweide, in die die Renntiere Gruben und Gänge gegraben haben" (Collinder I 14; III 81):

$\sim{ }^{*}$ ghas- o. ähnl. in ai. ghásati "verzehrt, verschlingt, isst" mit av. ga haiti „isst, frisst“", mit alterüml. Aorist ai. á-ghas usw., ohne ausserarische Verknüpfung, s. Mayrhofer, Aind. EW. I 358; Pokorny 452.

Das idg. *ed- „essen“ (Pokorny 287ff.) muss ein Konkurrent obiger Wurzel gewesen sein, doch war es augenschleinlich ein höflicherer Ausdruck als *ghas-; interessant ist es, dass gah-im Av. von daēvischen Wesen gebraucht wird oder von Tieren (hier das Kompositum niš-), s. Bartholomae, Altiran. Wb. 517. Auch im RV. usw. wird ghas- z.T. von den Tieren (Wölfen usw.) gebraucht. Daraus folgt, dass das indoiranische ghas- urspr. wohl nur "fressen“ von den Tieren bedeutete und kräftiger als *ed-war. Demnach stimmt es gut mit ural. Worte überein.

(26) finn.-ugr. *käte „Hand“ in finn. *käsi, Stam käte-, lapp. giettâ $\sim$ gie $\delta \hat{a}$,

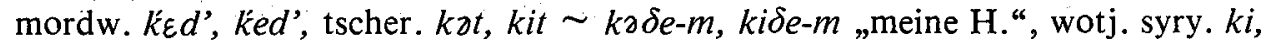
wog. $k \bar{a} t, k \bar{a} D$, ostj. kèt, kot, ung. kéz $\sim$ keze- (z.B. Szinnyei ${ }^{2}$, Nyelvh. ${ }^{7} 36$; Collinder I 87; III 81; Toivonen 263; Lytkin-Guljajev 123):

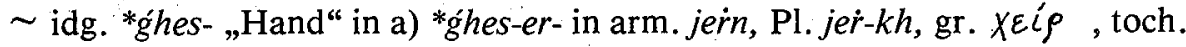
A $t$ sar, B šar, heth. keššar, keššer-; b) *g'hes-to- in ai. hásta-s, av. zasta-, apers. dasta-, lit. pa-žast-ẽ/ìs „Raum unter dem Arm, Achselhöhle“ (Pokorny 447). 
Schon früher veröffentlicht bei Verf., Die indouralische Sprachverwandtschaft und die indogermanische Laryngaltheorie, Slov. Akad. Zn. in Um. II, VII/5 (Ljubljana 1970), 35. Ural. + idg. auch bei Illič-Svityč 227, Nr. 80, wo noch drav. und semito-hamit. Material. S. noch Schlussfolgerungen 1.

(27) f.-ugr. *pitä- „(fest)halten usw.“ in finn. pitä- „halten; müssen, sollen“, mordw. ṕed'a „sich anschliessen, kleben; eigensinning anfangen“, ostj. pît- „geraten, fallen (irgendwohin); kommen; gefangen werden; münden (Fluss); anfangen“ (Steinitz, Vok. 60f., Nr. 333; Collinder I 108; FUV. ${ }^{2} 122$ usw.; doch lässt Toivonen $=$ SKES.583 das sonst hier angeknüpfte tscher. piסä- „binden“ und ung. füz-,schnüren, flechten usw.“ als eine eigene Gruppe ausser Spiel; ich fasste noch Dekl. 15 Nr. 6 alles Obige zusammen, jetzt schliesse ich mich SKES. an, zumal für tscher. und ung. Gruppe eine viel bessere idg. Verknüpfung zur Verfügung steht gr. psiathos):

$\sim$ idg. *pas- "fest“ Pok.789 in $t$-Bildungen arm. hast $<{ }^{*}$ pas-t $i$ - und anord. fas-t-r, ahd. fes-ti, fas-to. Ai. pastyám fällt weg.

Eine weitere, wohl parallele Etymologie bietet unten, Schlussfolgerungen Absatz IV Nr. (2), idg. *pĕеd- „fassen“, s. dort.

Regel Ila: Uralischem intervokalischem schwachem - $t$ - entspricht im Indogermanischen nach Ausfall des Vokals der ersten Silbe, wenn hinter Guttural geraten, das interdentale -p-:

(28) finn.-ugr. *puta $x$ + ein Suffix „Mastdarm“ in lapp. buttĕgě ds., ostj. puts „Dickdarm, Mastdarm (des Viehes, des Menschen)“ u.a. (Collinder 1 74):

$\sim$ ai.bhas-ád-F. „Hinterteil, weibliche Schamteile“ RV. usw., bha-sadyà- „am Hinterteil befindlich“ AV., bhấsada- etwa „Hinterbacken“ RV., VS. u.a., s. Mayrhofer, Aind. EW. I 489, wo die Lit. über bisherige Versuche, eine idg. Anküpfung zu finden.

(29) finn.-ugr. *kota „eine Wohnungsart“ in finn. kota „kegelförmiges Gebäude, vor allem als Küche; Zelt, Hütte“, koti, koto „Heim, Heimat" u.a., lapp. goatte

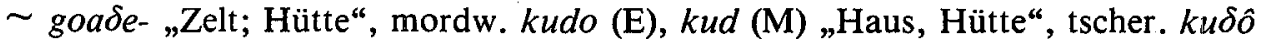
"die tscheremissische Sommerhütte" usw., wotj. kua, kua-la "Sommerhütte“, kor-ka „Haus“ (kor „Klotz, Block“), syrj. ko-la „Wald- od. Wiesenhütte (für Jäger und Mäher; aus Holz, das Dach aus Baum- und Birkenrinde usw.)“, ker-ka „Haus, Wohnhaus, Stube“ (ker „Balken“), ostj. kat usw. „Haus“, ung. ház ds., haza „heim, nach Hause“ (z.B. Szinnyei ${ }^{2} 32$; Szinnyei, Nyelvh. ${ }^{7} 36$; Collinder I 130f., III 82; Toivonen 224; Lytkin-Guljajev 114):

$\sim$ idg. * $k$ pei- „siedeln, sich ansiedeln, eine Niederlassung gründen“ bei Pokorny 626 in ai. kșéti „weilt, wohnt“, av. šaèiti ds., arm. šên „bewohnt, bebaut, Dorf“, gr. $\varkappa \tau \ell \zeta \omega$,gründe“, $\varepsilon \mathcal{U}-\varkappa \tau l-\mu \varepsilon \vee O S$,wohl gebaut, wohl bestellt", $\pi \varepsilon \rho \iota-$

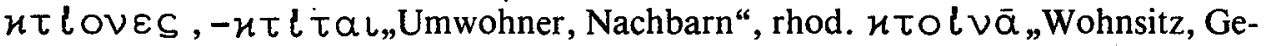
meindebezirk“. Vgl. auch Frisk, Gr. EW. II 34ff. mit Weiterem, vor allem aus dem Mykenischen. 
Zunächst muss man energisch den Satz von Pokorny, „gehört wohl zum Folgenden“, d.h. zur Wurzel *qbè (i)- „erwerben, Verfügung und Gewalt worüber bekommen", zurückweisen: das letztere hat einen Velar im Anlaut und ist eine schwere Basis; zu einer ural. Verknüpfung des letzteren s. Verf., Orbis XIX, 1970, 293 (finn.-ugr. *koçę- „halten usw.“).

Das fïnn.-ugr. *kota gilt in der uralistischen Literatur als eine Entlehnung aus dem Indoiranischen: aus av. kata- „Kammer, Vorratskammer, Keller“ u. Verw. (zum indoiran. Wort Pokorny 586f.); so z.B. Collinder 11. citt., Lytkin-Guljajev usw. Nur Toivonen a.a.O. erwähnt die iranische Hypothese nicht, man weiss nicht, ob aus Versehen oder weil er davon nicht überzeugt war.

Jedenfalls hat diese Herleitung aus dem Iranischen nichts für sich: die Sache, die *kota bezeichnet, ist von derjenigen, die av. kata- heisst, sehr verschieden. Ausserdem, und das ist das Wichtigste, existiert ein gleichlautendes altaisches Wort, das nach Räsänen 5 mit dem finn.-ugr. *kota identisch, d.h. verwandt ist: türk. kota „Haus, Zimmer", tschag. kotan "Viehstall", mong. kota, kotan "Zaun; alle Haustiere, die in einem Zaune vereinigt werden; Stadt, Stadtmauer", kor. kot "place, locality, site“. Dies Wort ist sicher nicht aus dem Iranischen entlehnt; damit fällt aber auch der iranische Ursprung für finn.-ugr. *kota!

Ist nun die Indigenität von *kota erwiesen, so ist auch idg. ${ }^{*} k p e i$ - ohne Schwierigkeit damit zu vereinigen: es ist ein denominatives Verbum wie *suei- bzw. *dhuěeiusw. oben unter Nr. 8 gegenüber ural. *ș̆upa bzw. çupa: das im Finn.-Ugr. auslautende $-a$ verwandelte sich in idg. $-e$-, daran trat das denominative Suffix $*_{-j}-$ (ursprünglich athematisch); die idg. Bedeutung dann etwa „ein Zelt aufstellen“, vgl. slvn. šotor-i-ti „kampieren“ von šotor "Zelt".

Das Wort *kota ist also ural.-alt.-idg. und muss eine ,primitive Wohnung, Zelt, Hütte“ bezeichnet haben.

\section{Suffixgleichungen}

Die uralischen Suffixe, die hier in Betracht kommen, haben die Struktur: $p$ oder $t+$ auslautender Vokal; im idg. schwindet der Reduktion zuliebe der auslautende Vokal, so dass das eigentliche Suffix nur in $-u$ - bzw. -s- (und eventuell - $p-$, dies hinter Guttural) besteht.

Regel I: Uralischem intervokalischem schwachem -p-entspricht im Indogermanischen $-\underline{-\mu}$-:

(1) ururalisches Suffix mit - $p$-für Adjektiva denominativa:

finn. $-p a,-p \ddot{a}$, nach unbetonter Silbe $-v a,-v \ddot{a}$, aus Systemzwang $v$-Form verallgemeinert: liha-va „fett, wohlbeleibt“ von liha „Fleisch“, väke-vä „kräftig, stark“ von väke- „Kraft" usw.; im Liv. erscheint noch die starke Form, $-p->-b-:$ ig $\bar{a}-b$ 
„langweilig“ = finn. $i k \ddot{a}-v \ddot{a}$ ds. von liv. $i g \bar{a}=$ finn. $i k \ddot{a}$ „Alter, Zeitalter, Lebzeit“; diese Adjektiva bezeichnen das reichliche Versehensein mit etwas;

mordw. $-v,-f,-\phi$, Adjektiva denominativa, die ein Behaftet-oder Versehensein mit et was bezeichnen: E salo-v, salu-v, $\mathrm{M}$ salu, Pl. salu-f-t "salzig“ von sal „Salz“;

wog. $-p,-p \dot{a}$, in der Regel als zweites Glied eines zusammengesetzten Attributs, seltener allein: $k \vec{a} t-s p, k a \bar{t}-p \dot{a}$,-händig“ von $k \bar{a} t$ „Hand“; bildet Nomin a possessoris oder drückt die Ähnlichkeit aus;

ostj. $-p,-\beta$, für Nomina possessor is usw., z.T. in possessiven Kompositis: von sem "Auge“ sēmə-p in DN əo-sēmə-p „einäugig“;

ung. (mit dem Auslautvokal des Stammes vereinigt) - $\bar{u}$, -ü (auch kurz): lábú, lábu „-füssig“ (z.B. négy-lábú vierfüssig“) von láb „Fuss“, nagy-fejü „grossköpfig“ von fö „Kopf“", alt noch -labo-v „füssig“;

nur Konglutinate im Sam.

Vgl. Szinnyei ${ }^{2} 88 f f$; Lehtisalo 244ff.; Collinder III 263.

$\sim$ idg. *-uo- in einigen Adjektiven, die haptsächlich ein Versehensein mit etwas bezeichnen:

ai. kēśa-vá- „langhaarig“ von kếśa- „Haar“, av. parna-va- „mit einer Feder versehen“ von parna- „Feder“, lat. ann-uo-s „(ein)jährig“ zu annus „Jahr“, cern-uo-s „kopfüber oder vorüber sich überschlagend" aus *kersn-e/ouo-s zu anord. hiarsi "Scheitel, Wirbel des Kopfes“ aus *kerson-; weiteres bei Brugmann, Grdr. ${ }^{2}$ II 1, 204ff.

Dies idg. *-uo- ist doch wohl eine Thematisierung aus älterem athematischem ${ }^{*}-u$ - (eig. ${ }^{*}-u o-=$ voruridg. ${ }^{*}-u_{n} e^{-}+x$, und dies $*_{-}^{*} e^{\prime}-=$ ural. ${ }^{*}-p a-$ ). Als Beispiel des athematischen Typus sei das Folgende angeführt:

Neben idg. *sal, * *sal-d, *sal-i steht auch *sal-u "Salz", s. Pokorny 878f., und zwar verbaut in gr. $\stackrel{\alpha}{\alpha} \lambda u-\varkappa \delta \varsigma$ „salzig“ (s. auch Frisk, Gr. EW. I 79); wie bei den Stoffnamen oft, kann auch hier eigentlich ein substantiviertes Stoffadjektiv vorliegen, *sal-u demnach Ntr. des Adjektives *sal-u- „salzig“ (auch toch. B salyiye „Salz“ kann auf einem *sal-iijo- „salzig“ beruhen); da nun idg. *sal „Salz“ auch im Uralischen (Finnisch-Ugrischen, s. Collinder I 137, wo nach anderen Entlehnung aus dem Idg. angenommen wird, ohne Grund!) vorkommt, finn. suola usw., ist es möglich, an Identität unseres *sal-u- „salzig“ mit mordw. salo-v, salu-v, salu ds. aus *sōla-pa zu denken. Freilisch ist es unmöglich, das Alter der mordw. Bildung zu kontrollieren, so dass es sich vielleicht nur um parallele Fälle handelt.

Anderes wird später zur Sprache kommen; hier erwähne ich nur noch idg. *u-ent- (Brugmann, Grdr. ${ }^{2}$. II 1, $461 \mathrm{ff}$., zum Heth. Kronasser, Etym. 266f.), das ebenfalls Versehensein mit etwas bezeichnete; ich habe darin eine schon indouralische Verbindung unseres $p$-Suffixes mit einem nít'-Element gesehen, ural. *-p-arǵce- (Lehtisalo 251f.) mit gleicher Funktion, s. Verf., Orbis XIX (1970), 312.

Man hat schon vor mir die Gleichung ural. - $p a-\sim$ idg. -40 - in denominativen Adjektiven aufgestellt (Anderson, s. Collinder, IU. 45f.). Seltsam ist dabei die Behauptung von Collinder: „Die Lautentsprechung fi.-ugr. $p: \beta \sim$ ie. $w$ kann nicht sonst erhärtet werden." Unsere Wortgleichungen Nr. (1) - (20) werden wohl dies zurückweisen können. 
(2) ururalisches Suffix mit ${ }_{-}^{*} p$ - für Nomina deverbalia:

finn. - $p a,-p \ddot{a}$ nach betonter Silbe, $-v a,-v a ̈$ nach unbetonter Silbe, bildet Partizipia I des Aktivs und des Passivs: hyvän suo-pa "bene cupiens, favens" von suo-,wünschen“, käy-pä raha „gangbares, gültiges Geld“ usw. von käy- "gehen“; tuo-va "holend" von tuo- "holen“, anta-va "gebend" von anta- "geben“, itke-vä "weinend" von itke- „weinen“; liv. iela $\bar{n}-b$ „lebend“ = finn. elä-vä ds. von elä- „leben" usw.;

wog. - $p$ Verbalnomen, u.zw. Präsenspartizip, z.B. $\beta i \gamma$-pả „nehmend“ von $\beta i \gamma_{-}$, auch Nomin a agent is, z.B. kǐš-p „Jäger“ von kins- „jagen, suchen“, intrans. $x o l-p$ „mortuus“ zu qol- „sterben“;

ostj. $-p$ usw. meistenteils in verselbständigten Substantiven, die die Handlung oder das Werkzeug der Handlung bezeichnen; das letztere wohl aus urspr. Nomina agentis: Trj. ïint $\hat{\jmath}-p$ usw. „Nähnadel“ von lïnt- usw. „nähen“;

ung. alt $-u$, sonst mit dem Auslautvokal des Stammes vereinigt zu -ó, $-b$, mundartlich auch -ú, -ü, für Verbalnomina und Substantiva deverbalia, z.B. in Gerundien I und II: $f u t-v a$, fut-ván "laufend" von fut „laufen“; im Auslaut $>$-ü: Leichenrede iaro-v $=/ j a \bar{r} o-u /$, „gehend" von jár "gehen“, heute járó ,gehend“, $k e ́-$ ró „,bittend“ von kér „bitten“, mundartlich fúrú, fúru „Bohrer“ von fúr „bohren“ usw.;

sar. jur. *-pa Nomina deverbalia, z.B. S pohō-pa „kraftvoll“ von pòhō „dem Ende nahe sein, nahen, der Genesung nahe sein" usw.

$\sim$ idg. $-u$ - und *-uo-in deverbalen Adjektiven, z.T. in der Bedeutung aktiver Partizipien; z.T. passiven Partizipien ähnlich:

ai. trş̣-ú- „lechzend“, got. paúrsu-s „dürr, trocken“, ahd. durri „dürr“, idg.

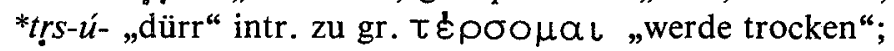

ai. ták-u- „eilend" neben tak-vá- „eilig, regsam“, got. pius = ahd. deo „Knecht“ (urgerm. *bey-wa-z) zu ai. ták-ti „eilt“;

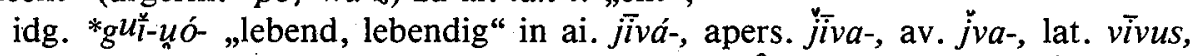
osk. bivo-, kymr. biw („Hornvieh“), lit. gývas, lett. dzîis, aksl. żivz; gr. B LOS „Leben“, got. qius „lebendig“, air. biu, beo, kymr. byw usw. ds. (Pokorny 468) von *gueiā $x$ - „leben“;

echt passiv:

idg. *tenH-ú-, *ténH-u-i $\bar{i}$ und ${ }^{*} t e_{n}$-ưó- „ausgedehnt, dünn" in ai. tanú-, tanvi

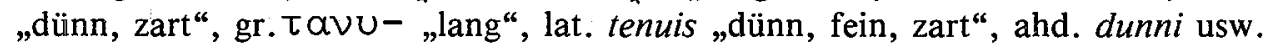
„dünn“, aksl. $16 n z-k 3$ ds.; gr. TavaFós „langgestreckt, lang“, air. tanae, bret. tanao „dünn“, lit. tévas, lett. tiêvs „schlank“ (Pokorny 1069) zu *ten- „dehnen, ziehen, spannen".

echt aktiv:

ai. grh-ú- „Bettler“, urspr. „ergreifend“, lit. grabùs „fingerfertig“ zu ai. grbh-, grh- „ergreifen“, lit. gróbti „raffen“.

Zum Ural. vgl. Szinnyei ${ }^{2} 77$ f.; Nyelvh. ${ }^{7} 84 f$ f; Lehtisalo 249ff.; Collinder III 270; zum Idg. Brugmann, Grdr. ${ }^{2}$ II 1, 176ff., $202 \mathrm{ff}$.

Auf beiden Seiten kann das nämliche Suffix a) aktive Partizipialien (von trans. und intrans. Verben), b) passive Partizipialien, c) Nomina actions, d) Werkzeugnamen bilden; schon alte Gleichnung, s. Collinder, IU. 45. 
Regel II: Uralischem intervokalischem schwachem -t-entspricht im indogermanischen $-s-$ :

(3) im Uralischen diente als Formans für die Bildung des pluralischen Stammes der Nomina u.a. auch $-t-$ :

finn. zu talo „Haus“ Nom Pl. talo-t, Stamm *talo- $\delta e$ - im Gen. Pl. *talo- $\delta e-n>$ taloin; zu hevonen Nom. Pl. hevose-t „die Pferde“, Stamm hevos-te- im Gen. Pl. hevos-te-n; kala Fisch", dazu Nom. Pl. kala-t, Gen. kalai-n aus *kala- $\delta e-n$; lapp. guole-t, guole- $k$ (-k aus ${ }^{*}-t$, vgl. die 2 . P. Sg. der Verba unten Nr. 5!) = finn. kala-t, von guolle „Fisch"; mordw. tolga-t von tolga „Feder", kal-t von kal- „Fisch"; wog. $x u l-t$ von $x u l$ ( $k u l$ usw.) „Fisch", $l \bar{u}-t$ von $l \bar{u}$ „Pferd“, àmpa- $t$ „Hunde“ von $\bar{a} m p$ „Hund“; ostj. kul-t von $k u l$ „Fisch“, laus-t von lau „Pferd“; sam. jur. $x a \overrightarrow{a l l e-v o n}$ $x \bar{a} l \bar{e}$ „Fisch“ usw.; urural. also *kala-t Nom. Pl. von *kala „Fisch“.

Vgl. Szinnyei ${ }^{2}$ 51; Ravila, FUF. XXVII (1941), 69ff.; Collinder III 297, § 925 :

$\sim$ idg. $-s$ als Zeichen des Plurals in verschiedensten Anwendungen:

a) im Kasussuffixe des Nom. Pl. des Genus animatum (Mask. und Fem.) -es: von *ped- *pod- idg. *pé/ód-es „Füsse“ in ai. páadas, arm. ot-kh, gr. $\pi \delta \delta \varepsilon \varsigma$, ags. fét, anord. føtr aus urgerm. *fótiz; von $r$-, $n$-Stämmen: *patér-es

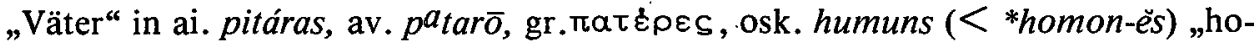
mines“, anord. dohtr-iR „Töchter", lit. móter-s u.a.; bei den $\bar{a}$ - und $o$-Stämmen Kontraktion in *-ās, *-ôs: idg. *ékūās, „Stuten" in ai. áśvās, osk. scriftas „scriptae“, got. gibōs „Gaben“, lit. rañkos „Hände“; idg. *ul $\iota^{\natural} q^{u} \bar{s}$ „Wölfe“ in ai. vŕkkās, got. wulfös, osk. Núvlanús „Nolani“. Zum Ganzen Brugmann, Grdr. ${ }^{2}$ II 2, $210 \mathrm{ff}$.

Die ständige vokalhafte Form des Suffixes -ĕs kann nicht lautgesetzlich sein, wenn man in - $e$ - urspr. auch im Indogermanischen auslautenden Stammvokal sehen will. Denn dieser Vokal, der z.B. im Gen. Sg. auf *-é-s, *-ò-s, weil betont, noch erhalten ist, s. unten Nr. 4, kann im Nom. Pl. nicht erhalten geblieben sein, denn hier ist das -ё- unbetont; dazu kommt, wie ich an einer anderen Stelle ausführen werde, noch die Ständigkeit der $e$-Farbe, die auch nicht zu erwarten wäre, wenn man in diesem -e-den Stammauslaut zu vermuten hätte. Die echte Form des pluralischen Stammes, der mit uralischem Typus auf $t$ gänzlich parallel wäre, ist nun im Lok. Pl. erhalten, s. unten b); im Nom. Pl. auf -es muss man eine Analyse in -e-s vornehmen, wo nur $-s$ das wahre Pluralzeichen ist, das - $e$ - aber in meinem Flexionsbuch zur Sprache kommt. Vgl. meine Dekl. (1975), SS. 33 und 35-38.

b) im Kasussuffix *-su des Lok. Pl., s. Brugmann, Grdr. ${ }^{2}$ II 2, 247ff. und vgl.: idg. *ékūa-su „in Stuten“ = ai. áśvāsu, lit. rañko-su in Händen“; ai. pat-sú „auf den Füssen“ usw.

Diese Endung ist in pluralisches $-s$ - + Lokativformans $-u$ - (worüber noch in meinem Flexionsbuch) zu zerlegen; das erweist sich nicht nur durch das Bestehen des gr. Formans - $\sigma \iota$, z.B. altatt. $\delta l u \eta-\dot{\sigma} \iota$ „Rechten“ oder myken. do-e-ro-i

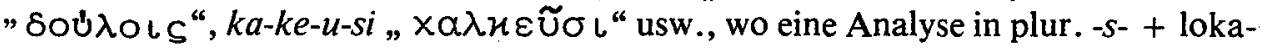
tivisches $-i$ die weit überzeugendste ist, sondern vor allem durch die archaische Form des Lok. Pl. von *pĕed- M. „Fuss“: *pēd-s „auf den Füssen, bei den Füssen“ und „zu Fuss“ in lit. pěs-čias „zu Fuss“ = *péds- "auf den Füssen“ + Suffix *-tịo- (unan- 
nehmbar die Urform *pēd-tio-), sl. pěšc „zu Fuss gehend“ = Lok. Pl. *pēds „zu Fuss" + Suffix *-io- (oder *-io- ,gehend“); normalisiert ist dieser Lok. Pl. im Adverb *pētsu in alb. për-posh „unten“, air. $\bar{s}$,unterhalb“ (vgl. Pokorny 790; Vasmer, Russ. EW. II 353; Fraenkel, Lit. EW. 562 mit z.T. unwahrscheinlichen Deutungen). Vgl. Dekl. 36.

Der Lok. Pl. *pēd-s ist eig. nur ein Pluralstamm, entstanden aus einer zweisilbigen Form *pĕdĕ-s; hier schwand das zweite -e- (unter der Dehnung des ersteren - $e$-), das sicher $=$ urspr. Stammauslaut von *pëd-ist (vgl. Gen. Sg. *pedé-s usw.) und also von jeher kurz war, also reduktionsfähig (im Gegensatz zum Nom. Pl. auf *-e-s). Als Lok. Sg. fungiert bekanntlich sehr oft der blosse Stamm; dasselbe ist für die ursprüngliche Pluralflexion anzunehmen: als Lok. Pl. muss demnach der blosse Pluralstamm fungiert haben, und dieser ist gemäss obigen ural. Vergleichen mit $-s=$ ural. - $t$ ausgestattet worden. Erst nachträglich wurde der Lok. Pl. mit echt lokativischen Mitteln $(-i,-u)$ verdeutlicht.

c) in den pluralischen Stammformen der Pronomina personalia der 1. und 2. Person:

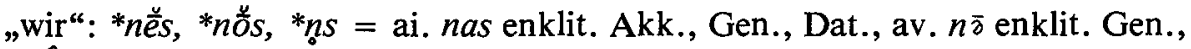
Dat., nå enklit. Akk., jungav. nō enklit. Akk., Gen., Dat.; alb. na proklit. Akk., Dat., ne haupttoniger Akk., Gen., Dat.; lat. nōs; got. Dat., Akk. uns usw. (Brugmann, Grdr. ${ }^{2}$ II 2, 408f.);

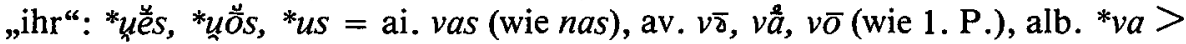
$u$ proklit. Akk., Dat.; lat. vōs; usw. (Brugmann ibd.).

Es ist schon Brugmann 408 klar gewesen, dass „Diese Formen ohne Kasuszeichen sind, da $-s$ als Zeichen des pluralischen Sinnes zu gelten hat." Das beweisen die

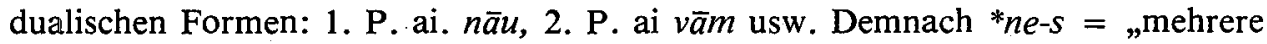
Ich", *ue-s = „mehrere Du“.

d) als einmal das $-s$ im Nom. PI. *-e-als eine Art bewegliches Pluralzeichen (wegen des Vergleiches mit Du. $-\overline{\bar{e}})$ aufgefasst wurde, konnte es auch auf andere Kasusformatien, z.T. auf solche ganz jungen Datums, übertragen werden, um sie zu pluralisieren:

Akk. Pl. Mask. und Fem. ${ }^{*}-n-s=$ Sg. $-m+$ pluralisches $-s$ (Beispiele bei Brugmann a.O. 220ff.);

Dat.-Abl. Pl. *bh(i)o-s, *-mo-s, -mu-s (Brugmann a.O. 257ff.); 262ff.).

Instr. Pl. *-bh $\ddot{i}-s, *_{-} m \bar{l}-s$, wohl auch $*_{-} \bar{o} i-s$ und $*_{-} \bar{i}-s$ usw. (Brugmann a.O.

Hier zeigt der Vergleich mit singularischen Formatien Instr. *-bhi, *-mi (bei Brugmann a.O. 186ff.) sowie mit dualischen Kasuszeichen Dat.-Abl.-Instr. *bh(i)ēm, *-mā (bei Brugmann a.O. 203ff.), dass das auslautende $-s$ ein abtrennbares Element mit deutlicher Pluralfunktion ist.

Die Pluralfunktion des Lautes $*_{-s}$ in den behandelten Kasuszeichen war schon lange bekannt, s. Brugmann a.O. 120. Vgl. noch Dekl. 35-38!

(4) im Uralischen diente als Endung des Partitivus Ablativus die Silbe -ta:

finn. luo-ta „von“ (Lok. luo-na „bei“), al-ta „unter...weg od. hervor" (von ale„das Untere“), Partitiv. talo- $a$ „vom Haus“, vet-tä „Wasser, de l'eau“ (Stamm 
vete-); lapp. N vuol-de „unten, von unten“ (zu ale- oben); mordw. al-da, al-do "unter...weg od. hervor“ (al- „das Untere“), ked'e-d'e „von einer Hand“ (von ked');

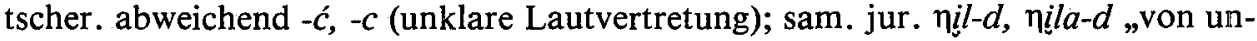
ten..." (zu finn. ale-) usw.

Vgl. Szinnyei ${ }^{2}$ 56-58; Collinder III $287 \mathrm{ff}$.

$\sim$ idg. Kasussuffix $-s$, -és, -òs, das als Genitiv- und Ablativendung dient: idg. *pe/od-és und *-òs, „des Fusses“ und „vom Fusse“ in ai. padás, gr. no lat. pedis usw. S. Brugmann, Grdr. ${ }^{2}$ II, $150 \mathrm{ff}$.

Vgl. meine Dekl. 50-52.

Die kürzere Endung idg. $-s$ kommt hinter Vokalen und Diphthongen vor, vgl. gr . $\vartheta \varepsilon \tilde{\alpha}-\varsigma$ „der Göttin“, lit. mergõ $s$ „des Mädchens" usw., ai. noch in gnấ-s-(páti-„Gemahl eines göttlichen Weibes"); ai. matế-š „des Sinnes“, osk. aetei-s „partis“, got. anstai-s „der Gunst“, lit. naktiē-s „der Nacht“; ai. sūnó-š „des Sohnes“ = lit. sūnaũ-s, got. sunau-s, osk. castrou-s „fundi“; aber fakultativ auch bei $n$ -

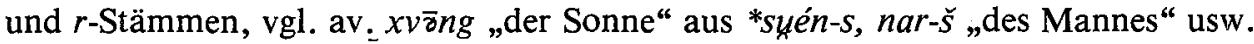

Es ist nun sicher, dass das $-e-,-o-$ der idg. längeren Endung zum Stamm gehört und erst dann, als diese Vokale in unbetonter Stellung, z.B. im Nom. Sg. ( $\left.{ }^{*} p \bar{e} d-s\right)$ oder im Akk. Sg. (*péd-m) schwanden (voruridg. *pěde-s, *péde-m), durch die nun natürliche Analyse in konsonantischen Stamm + Endung, der letzteren zugerechnet wurde; die kürzere Endung dagegen zeugt von altem indoural. *-ta in ursprünglicher Form; hinzugefügt sei, dass wenigstens die idg. $n$ - und $r$-Stämme von Haus aus ebenfalls vokalische Stämme (auf urspr. -ne-, -re-) gewesen sind, dass sie aber vor der Gen.-Abl.-Endung *-ta $\left(>-b e>*_{-s}\right)$ teilweise ohne die Wirkung des Ablautes ihr - $e$ - abwarfen, wie das auch einige finnische Stämme vor -ta, -tä tun, vgl. oben vet-tä und tytär-tä von tytär "Tochter" (Stamm tyttäre-z.B. im Gen. Sg. tyttäre-n). Hier kann man mit einer alten Wechselfähigkeit der indogermanischen sowie uralischen Stämme rechnen, die für die idg. Kasusendungen z.T. von grosser Bedeutung war. Vgl. Dekl. 92-95; Lingu. XIV (1974) passim. Usw.

Die syntaktische Verwendung des idg. Gen.-Abl. Sg. auf $-s$ usw. (darüber Brugmann, Grdr. ${ }^{2}$ II 2, 565ff., 494ff.) zeigt so grosse Ähnlichkeiten mit ural. PartitivAblativ auf -ta (kurze Skizze bei Collinder III 287ff., $\$ \S 880-884$ ), so im partitiven Gebrauch, als Separativ (Ablativ in konkretem Sinne), Partitiv/Ablativ des Vergleiches usw., dass ein Zweifel an der historischen Zusammengehörigkeit beider Endungen ganz unsinnig ist. Es liegt an der Hand, dass nun unser Vergleich manches Problem um die Verwendung des Gen.-Abl. Sg. lösen wird.

Die letzteren Bemerkungen sind nun auch darum von Gewicht, weil man bisher die ural. Endung -ta des Partitivs-Ablativs mit idg. $-t$ der Ablativ-Sg.-Endung der $o$-Stämme (*ékuōt/d „vom Pferde“", Brugmann, Grdr. ${ }^{2}$ II 2, 163ff.) zu vergleichen pflegte, vgl. Anderson bei Collinder, IU. 22f.; Jensen, Hirt-FS. II 178; Illič-Svityč 11, 213 u.a. mehr. Nun ist aber die Endung idg. $* \tilde{\bar{e}} / \tilde{\bar{o} t} / d$ mit der finn.-ugr. Endung des Abessivs *-kta(-k) zu identifizieren, wie ich in meiner Arbeit Die indouralische Sprachverwandtschaft und die indogermanische Laryngaltheorie, Slov. Akad. Zn.

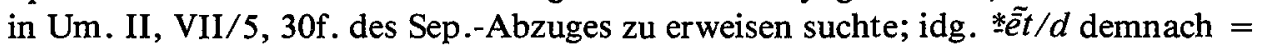
voruridg. *-é-Hte aus indoural. **-é-kta. Wohl aber ist zuzugeben, dass das schliessende $*_{-} t$ der eben genannten Endung mit dem indoural. ${ }^{*}$-ta des Ablativ-Partitiv 
identisch ist. Doch zwischen Vokalen muss - $t$ - der Endung -ta im Idg. durch $-s$ vertreten sein und so ist die Gleichstellung von Anderson im allgemeinen zu verwerfen. Ein Rest ihrer Berechtigkeit wird unten in den Schlussfolgerungen IV. zur Sprache kommen.

(5) als Endung der 2. P. Sg. wurde im Uralischen ein - $t$ gebraucht: finn. mene-t „du gehst“, men-i-t „du gingst“; mordw. kula-t „du stirbst“, ṕel'-i-t „du fürchtetest"; tscher. ula- $t$ "du bist“, kola-t "du hörst“; ung. ("schwache Stufe" * $*_{-} \delta$ ?) alszo-l "du schläfst“ usw.; lapp. $-t>-k$ (wie im Pluralstamm, Nr. 3): uole-t $=$ finn. paia-t „du brennst“. (intr.).

Vgl. Szinnyei ${ }^{2}$ 129; Collinder III 308 und 310:

$\sim$ idg. $-s$ als Sekundärendung und $-s-i$ als Primärendung der 2. Sg.: idg. * ${ }^{*}-s t \bar{a}-s$

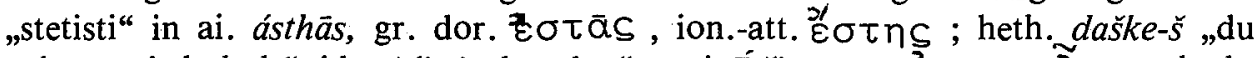
nahmst wiederholt"; idg. *éi-si „du gehst" $=$ ai. $\bar{e}-\breve{s i}=$ gr. $\varepsilon \hat{\imath}$ aus $\star \varepsilon \widetilde{\imath}-\sigma \iota$, heth. $p \bar{a} i$-ši $=$ *bhe-ei-si usw.; dazu die medialen sek. -so, primär -soi, vgl. zu allem Brugmann, Grdr. ${ }^{2}$ II 3/2, 603ff. und 644ff.

$\mathrm{Zu}$ den $t$-Endungen in der 2. Sg. und zum Verhältnis zu den Endungen der 2. Pl. Akt. s. unten Schlussfolgerungen, Pkt. II!

(6) ural. Suffix mit - $t$ - für Nomina deverbalia, und zwar in Infinitiven und Partizipien:

finn. In finitiv (mit Kasusendugen, lativ. -' und translativ. -kse- + Possessivsuffix), mit starken Lautveränderungen: nach $-s-$-ta-, -tä-: juos-ta „laufen “ $<*$-ta- $k$, sonst z.B. teh-dä "tun“, men-nä ,gehen“ aus *tek-tä-k, *men-tä-k usw.; sano-a „sagen“ aus *sano- $\delta a-k$ u.a.;

lapp. Infinitiv N gullâ-t ,horen“ usw.;

mordw. koma-do, koma-da "gebückt, in gebückter Stellung“ vom Stamm koma- usw., Verbalnomina ohne Kasusssuffix, als Adverbien gebraucht;

ostj. Infiniti: DN $m a n-D \dot{a}, \mathrm{Kr} . m a n-t \dot{a}, \mathrm{~V} m a n-t a ̈, \mathrm{Vj}$. man-tä, Likr. man-

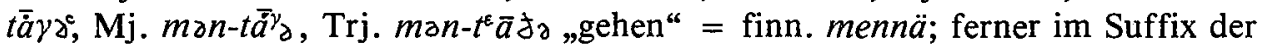
Participia Präs., z.B. ĭănt-ti ,spielend“ von ĭănt- „spielen“; im passiven Partizip Präs., im passiven Partizip Prät. usw.

Weitere Verwandte im Wog., Ung., Sam.

S. Szinnyei ${ }^{2} 79$; Szinnyei, Nyelvh. ${ }^{6}$ 86f.; Lehtisalo 273ff.; Collinder III 271:

$\sim$ idg. $-s$ - als Hauptmerkmal der verschiedenen In finitive: a) auf ai. $-a s-\bar{e} z . B$.

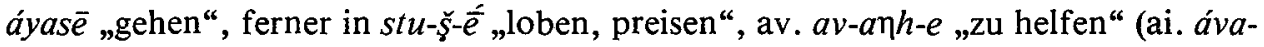
$s \bar{e})$; b) lat. -ere z.B. in vehere „fahren“ aus *ueǵh-es-i; c) gr. (sekundär aoristisch ge-

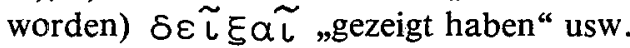

Vgl. Brugmann, Grdr. ${ }^{2}$ II 1, 525, $\S 400$; Sommer, Hb. ${ }^{2-3} 519 f$.; Schwyzer, Gr. Gr. I 808.

$\mathrm{Zu}$ beachten ist, dass sich z.T. perfekte Gleichungen zwischen Ural. und Idg. aufstellen lassen, die sowohl die gleiche Verbalwurzel sowie unseren Infinitivstammauslaut enthalten:

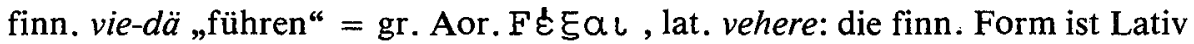

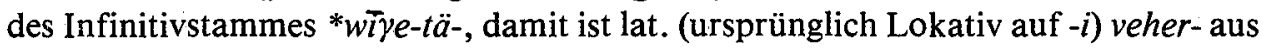


*ueǵh-es- identisch, noch mehr aber das gr. *ueǵh-s-a-i, denn dies ist eine Erweiterung des ehemaligen Lativs auf $*-a-H(+$ deiktisches $-i)$, demnach vorurfinn. ${ }^{*}$ wi ye$t \ddot{a}-k=$ gr. *ueǵh-sa-H. Mehr in meinem Flexionsbuch.

(7) ural. Suffix mit $-t$ - für Verba causativa deverbalia (Lehtisalo 294ff.): finn. nur nach einem auf Konsonanten ausgehenden Stamm, z.B. pääs-tä- „loslassen, gehen lassen, weglassen, von sich lassen, befreien “ von pääse- „loskommen, entgehen, befreit werden“; nach dem Suffix -ne-: ojen-ta- „gerade machen“ zu oikene- ,gerade werden“ usw.;

lapp. $\mathrm{N}-d i-t$, an die starke Stufe intransitiver $\hat{a}$-Verben und $o$-Verben angefügt: duttâ-di-t „zufriedenstellen, befriedigen, stillen“ von duttâ- „zufrieden werden mit, befriedigt werden durch";

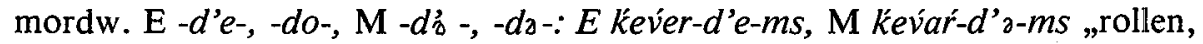
wälzen“ zu E kevére-ms, M kev́rrə-ms „rollen; sich wälzen, sich herumdrehen “ usw.

tscher. -te- usw., z.B. KB $\beta a l-t e-m$, U $\beta$ ol-te- $m$,herabsenken, hinablassen, fallen lassen, schütten“ zu KB $\beta a ̀ l e-m$, U Bolè- $m$,herabsteigen, herunterklettern; herabsinken, untergehen" u.a.;

syrj. -ed-, -ett- z.B. in kus-ed- „löschen, auslöschen“ (trans.) zu kus- „auslöschen, intr." u.a., ähnlich wotj. -et-;

Reste im Wog., Ostj.; Verwandtes im Sam.:

$\sim$ idg. $-s$ - als Formant kausativer deverbaler Verba und verbaler Systeme; ich nenne nur:

toch. Klasse VIIIb, Präsentia mit $-s$ - kausativer Bedeutung: neben $p$ olk- „brennen“ (intr.) (Präs. III B pəlketər) steht das Kausativum B 3. Sg. polk-š-ən „,brennt (trans.), quält", in A pəlk-s-eñč; vgl. Krause, Westtoch. Gr. I 79ff.; KrauseThomas, Toch. El. I 207ff.;

sonst etwa noch av. vax-š-aiti „lässt wachsen“, ai. vák-ṣ-ana-m „Stärkung“, gr.

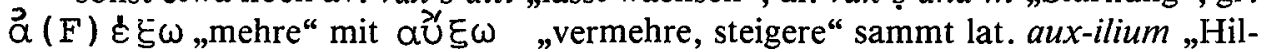
fe“, urspr. „Verstärkung“, von Adj. *aux-ilis „zur Verstärkung dienend“, also idg. Kausativum *aueg-s-, *ueg-s-, *aug-s- „vermehren, wachsen lassen, stärken“ zum intr. *aueg- „sich vermehren, zunehmen“; vgl. Pokorny 84f.; Walde-Hofmann, LEW. $i$ I 89; Frisk, Gr. EW. I 188 usw.

Die Funktion ist demnach auf beiden Seiten die gleiche.

(8) ural. Suffix mit $-t$ - für Substantiva denominativa (Lehtisalo 264ff.), z.B. in:

finn. $-u t-\sim-u e-\left(<*_{-}^{*} u \delta e-\right)$ usw. in Deminutiven, z.B. marj-ut zu marja „Bee-

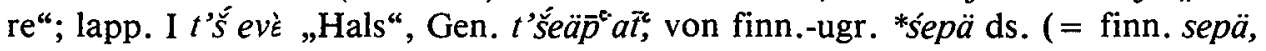
sepi) usw.;

tscher. z.B. nulgu-do „Weisstanne“ zu nulyo ds.;

Unsicheres im Sam.:

$\sim$ idg. *-(e)s- in gewissen Wörtern, wo es einfach der Erweiterung ohne irgenwelchen Bedeutungsunterschied dient, z.B. in:

idg. *qreuə-s- und ähnl. „dickes, stockendes Blut; blutiges, rohes Fleisch“ (Po-

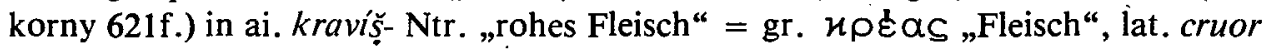


M. „das rohe, dicke Blut“, *qrû́z-d- „dick machen“ in ai. krū-d-áyati „macht dick, fest", av. xrǚž-d-ra- „hart" usw. zu idg. *qreuə-, q́ruH- „dickes, stockendes Blut" in ai. kravyam „Blut" = lit. kraũjas ds., mir. crū „Blut" = av. $x r \bar{u}-\mathrm{F}$. „Stück blutiges Fleisch“, sl. kry, krø̋ve „Blut“, lat. cru-entus „blutig“ zu av. xrvant- „grauenhaft, grausig" usw.;

idg. *Kerð-S-, *kra-s- „das Oberste am Körper: Kopf, Horn; Gipfel“ (Pokorny 574f.) z.B. in: ai. síras- nur Nom: Akk. „Kopf, Spitze“, av. sarah-„Kopf“, gr.

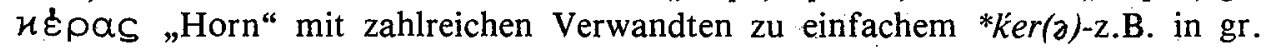
นdo „Kopf“ ù.a.;

idg. *uet-es- Ntr. „Jahr“ bei Pokorny 1175 in ai. vat-s-á- M. „Jahr“ = luw. $u \check{s ̌ a}$ - und hgl. usa-ds. (idg. *uet-s-ó- nach Verf., Lingu. IV (Ljubljana 1961),

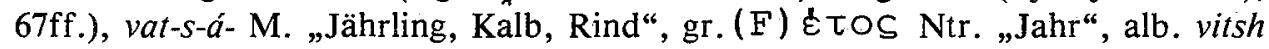
„Kalb“ (richtig viç) aus *uet-es-o-, messap. ata-vetes „vom gleichen Jahr", lat. vetus

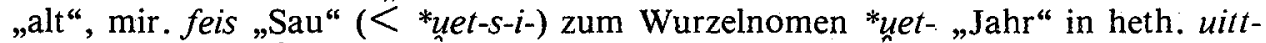

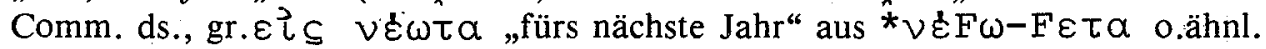
vgl. Frisk, Gr. EW. II 313), dazu idg. *pér-ut(-i) Lok. Sg. „im vergangenen Jahre“ (s. Pok. a.a.O.).

Auch im Idg. kann eigentliche Bedeutung des Suffixes irgendwie deminutiv gewesen sein. Freilich ist idg. $-s$ - in solcher Stellung mehrdeutig, zur Konkurenz mit ural. *-kse-, ebenfalls deminutiv, s. Verf., Acad. Sc. er Art. Slov. II, VII/5 (Ljubljana 1970, 213f. (= 31f. des Sep.-Abdr.).

(9) finnisch-ugrisches Suffix mit - $t$ - für Adjektiva denominativa (Lehtisalo 270f.):

finn. kolea $<{ }^{*} k o l e \delta a$ „uneben" zu kole „unebene Stelle“, válkea $<{ }^{*}$-e $\delta a$ weiss" zu valkoinen ds., valo „Licht“, weps. vâuğge-d, Pl. våuk-ta-d „weiss“ usw.; s. unten Tscher.!

Konglutinat -ut- (deminutiv) z.B. in finn. ohu-t „dünn“ zu ohkanen ds.;

lapp. $\mathrm{N}$ usw. $-d-$, bildet Adjektive, die den Besit $z$ dessen ausdrücken, was das Grundwort angibt: čuowgâ-d „hell, klar“ von čuowgâ „Licht“ usw.;

mordw. E -do, $\mathrm{M}$-dă für Adjektiva denominalia: $\mathrm{E}$ val-do, $\mathrm{M}$ val-dă "hell, licht", vgl. finn. valea, vaalea „bleich" zu valo "Licht";

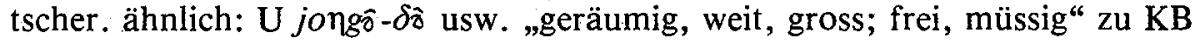
jongว̀ „Geräumigkeit, Gelass, offener Plàtz, freie Zeit, Musse“ usw.; mit finn. valkea identisch: KB $\beta a l \gamma \hat{\partial}-\delta \hat{\jmath}$ „weiss“ usw., s. auch mordw. val-do ua.;

wahrscheinlich syrj. -id, P PK -it und wotj. -it, -it: syrj. kuri-d, P PK kuri-t „bitter, scharf schmeckend; Bitterkeit“, wotj. kuri-t usw. ds. zu syrj. kur-di „bitter werden", Grundwort ein Nomen *kur? Zur Phonetik Lehtisalo 272f.;

im Sam. unsicher:

$\sim$ idg. *-(e)s- in einigen Nomina, die urspr. Adjektiva waren und möglicherweise von nominalen Grundwörtern abgeleitet sind. Statt eine Fülle unsicherer ad-

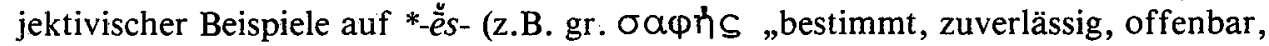
deutlich, klar" zu Adv. ođ $\varphi$ a „bestimmt, sicher, zuyerlässig“ bei Frisk a.O. II 684) anzuführen sei es mir erlaubt, auf ein wahrscheinlich finn.-ugr.-idg. gemeinsames Geschöpf dieser Art hinzuweisen: 
bei Collinder I 117 findet man eine Gruppe, die ich wegen des Perm. auf ein urfinn.-ugr. *ćara $x_{-}$„trocken; trocknen“ zurückführe: ung. szára-d- „trocknen, trocken werden“, tscher. sər ok „trocken, ausgedörrt", wotj. ćirs, cirs, ćeres „sauer, bitter“, syrj. cir- „einen Stich bekommen (Fisch, Speck), abstehen, herb, sauer werden (Bier), bitter, herb werden; heiser werden“, ćirem jai „,in der Sonne gedörrtes Fleisch“ usw.; wog. šurr-, sur- „trocken, dürr werden“, ostj. sar- usw. (Karjalainen II 877) „trocken werden, trocknen, austrocknen“" (Fisch, Wasser usw.):

idg. *ter-s- „trocknen, verdorren; Durst, dürsten“ bei Pokorny 1078f., z.B.

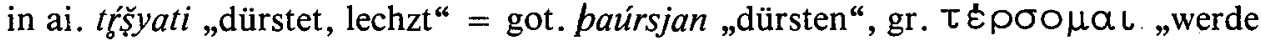
trocken“ u.a., woneben nominal *teres- „trockenes Land“ in lat. terra „Erde“, osk. terúm, teer[um] „territorium“, teras „terrae“ (urital. *tērs-), dazu *tēes- Ntr. in air. tīr Ntr. „Gebiet“, korn. bret. akymr. tir „tellus“, air. tïr-im „trocken“, demnach idg. (ital.-kelt.) *têr-es-.

Der neutrale es-Stamm *tēr-es steht in genetischem Zusammenhang mit dem verbalen *ter-s; die Wurzel *ter-ist mit ural. (finn.-ugr.) *carax-identisch, das esSuffix (-e- urspr. Teil der Wurzel!) kommt aber wieder zum Vorschein in ung. szára- $z$ „trocken, dürr". Es ist zwar an der Hand der idg. Gegebenheiten sowie aus Lehtisalo 280 (száraz unter den Bildungen mit ural. - $t$ - für Nomina dever balia) anscheinend zu vermuten, dass auch idg. $-s$ - hier deverbal ist, jedoch ist ung. szára- $z$ sehr ähnlicher Bildungsart wie finn. Typus auf $-e a<*_{-}^{*} e \delta a$ oben. Man kann demnach auch hier ein denominales idg.-finn.-ugr. *t'arax-ta „trocken“ sehen. Für solches Alter der Bildung spricht die Isoliertheit auf der ungarischen Seite (vgl. auch Szinnyei, Nyelvh.i 86 unten).

Aber auch mit Annahme deverbalen Charakters kommt man aus, denn auch Fälle wie finn. kolea, valkea sind nicht ganz rein denominal.

(10) hinter Guttural kann man im Idg. eine kombinatorische Variante des $-s$ - erwarten, nämlich ${ }^{*}-b$-; das steckt tatsächlich in folgendem idg. Verbalstamm:

*tekp- „zimmern“ (zum Bedeutungsansatz s. gleich unten) bei Pokorny 1058f.

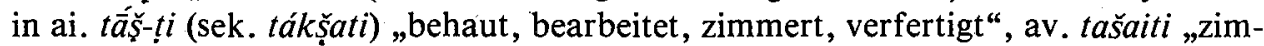
mert, schneidet zu, verfertigt"; lit. tašaũ, -ýti „behauen", lett. tešu, tèšu mit Inf. test, tèst ds., aksl. tešg, tesati ds.; idg. *tekpō(n) „Zimmermann“ = ai. tákș̆an-, gr. $\tau \varepsilon \mathcal{H} \tau \omega \nu$ usw. Fern muss lat. texō „flechten, weben“ bleiben, da bedeutungsgeschichtlich unvereinbar mit den Obigen (Pokornys „flechten, das Holzwerk des geflochtenen Hauses zusammenfügen" für die idg. Wurzel *tekp-ist ad hoc konstruiert), ebenso heth. takkeš-, takš- „zusammenfügen, unternehmen“, worüber allein richtig Laroche, BSL.58 (1963), 71 (idg. *dek- bei Pokorny 189ff.).

Das *- $b$ - dieser Wurzel kann kaum als echter Wurzelbestandteil betrachtet werden; viel eher ist es ein Suffix, das auf indoural. *-t-zurückzuführen ist, somit etwa mit dem idg. *-s- und ural. *-t- der Verba ca usativa deverbalia der Gruppe (7) oben identisch sein wird, obwohl im Idg. *tek- $b$-die kausative Funktion schon ganz verloren hat. Aber auch in Nr. (7) hat man derartige Prozesse beobachten können.

Die uralischen Sprachen bieten vorläufig nichts zur Vergleichung mit dem Vorsuffix-Stück *tek-, wohl aber das Tungusische: ewenk. čikā- „abscheiden, ab-, 
niederhauen“, über dessen eventuelle nostratische Verwandtschaft (nur Kartwelisch) bei Illič-Svityč 210 , Nr. 55. Viel geeigneter ist nun die idg. Wurzel *tek-. Man muss annehmen, dass das Wort ursprünglich onomatopoetisch war, mit anlautendem *čc-(ć-? oder $\breve{c}-$-?), auf der indogermanischen Seite jedoch dessen Lautstruktur normalisiert wurde, wie sonst oft, vgl. meine Indouralica XIII.

\section{Schlussfolgerungen}

Die oben als Grundlage für Etymologien und Suffixgleichungen benutzten zwei phonetische Entsprechungsregeln:

a) ural. schwaches intervokalisches $-p-=$ idg. $-u$-,

b) ural. schwaches intervokalisches $-t-=$ idg. 1. normal $-s-$, 2. nur hinter Guttural $-b$-,

müssen noch weiter besprochen werden, um in einen richtigen Rahmen eingesetzt zu werden und somit an Sicherheit zu gewinnen.

\section{Forschungsgeschichte.}

Zuerst wurde von mir, schon vor Jahr 1950, entdeckt, dass einem uralischen schwachen intervokalischen - $k$ - im Idg. der sog. „Laryngal“ - $H$ - entspricht; die erste selbständige Etymologie auf diesem Gebiet machte ich mittels finn.ugr. *teke- „tun, machen“ (heute bei Collinder I 119 u.a.) = idg. *dheH- „setzen, stellen, legen“ und „tun, machen“ (Pokorny 235ff.), woraus *dhè- usw. Diese Etymologie habe ich Prof. Oštir mitgeteilt, der sie in laryngalistischem Rahmen ausgenutzt hat.

Auf dieser Entsprechungsregel weiterbauend, vor allem überzeugt, dass das idg. $-H$ - ein Spirant war (wohl mehrere Arten echter oraler Spiranten, parallel mit idg. drei Gutturalarten, worüber an anderer Stelle), machte ich bald nachher neue Entdeckungen auf dem Gebiete der Entsprechungen der ural. schwachen Verschlusslaute. Ich stellte fest, dass viele ural.-idg. Gleichungen möglich und sehr überzeugend sind, wenn man auch für ural. schwache Verschlusslaute in intervokalischer Stellung auf der indogermanischen Seite Spiranten als Entsprechung aufstellt; so wurde die Regel b) (ural. $-t-=$ idg. $-s$-) entdeckt. Etwas später fügte ich hinzu die Regel a) (ural. $-p-=$ idg. $-u_{-}$-), denn hier musste man einen Gedankensprung machen, da im Idg. echter Spirant bilabialer Art (- $\left.\varphi_{-}\right)$oder ein eng verwandter Laut (labiodentales $-f$-) bekanntlich fehlt. Ein weitere Korrektur zur Regel b) (ural. - $t$ - $=$ idg. - $\mathrm{p}$ - hinter Guttural) machte ich einige Jahre später.

Kurz habe ich die eben dargelegten Entsprechnungsregeln schon in meiner Arbeit Die indouralische Sprachverwandtschaft und die indogermanische Laryngaltheorie, Acad. Sc. et Art. Slov. II, VII/5 (Ljubljana 1970) besprochen. Diese Arbeit galt der Regel ural. $-k-=$ idg. $-H-$. Jedoch musste diese Entsprechungsregel in einen weiteren Rahmen eingesetzt werden und so sprach ich a.O. S. 217 (= S.35 des Sep.Abdr.) auch von Regeln ural. $-p-=$ idg. $-u_{n}-$ und ural. $-t-=$ idg. $-s-$. 
Für ural. $-p-=$ idg. $-u$ - führte ich dort an:

a) finn.-ugr. (eig. nur ugr.) *sip $\left(a^{x}\right) k a^{x}$ - ,saugen“ in wog. sip $\gamma_{-}$, ung. sziv-, szip- usw. (Szinnyei, Nyelvh. ${ }^{7} 153$ ):

$\sim$ idg. *sū-, *seus - „saugen“, auch „schlürfen“ (bei Pokorny 912f. Vermischung mit gleichgebauter Wurzel m. d. Bed. "Saft, Feuchtes“) in lat. $s \bar{u}-g-\bar{o}$ „sau-

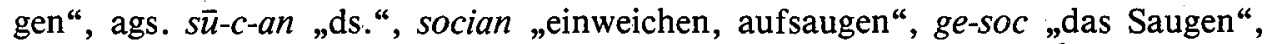
anord. $s \bar{u}-g-a=$ ahd. $s \bar{u}$-g-an „saugen“, lett. $s \grave{u}-k-t$ „saugen“; ai. $s \bar{u}-p-a-$ „Brühe,

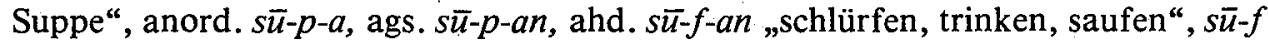
„Brühe, Suppe“, vollstufig sou- $f$ "Suppe“ usw.

Diese Etymologie fusst auf der Gleichsetzung von ural. *sipa $x_{k a} x_{-}$(daraus später *sipkax-) mit idg. ${ }^{*}$ seua $x_{H}$-.

S. auch a.O. S. 20 des Sep.-Abdr.

b) ebenda steht die Gleichung:

ural. *tupka "Schopf, Haarschopf" in finn. tukka "capronae frontales pendentes e.c. equi, inde crinis" = "Stirnhaar, Haupthaar“, karel. tukka „Haar" usw., tscher. tupka „Flocken Wolle oder Werg“, „das Haar des Menschen“ usw., syrj. tup-jur „Eule“ (jur = „Kopf“, tup-jur demnach „Schopf-Kopf“), tup-jurà „schopfig“; ?sam. jur. tupkāpt, tipkâpt „brillenartiger Schutz vor den Augen“ (Setälä, FUF. VI (1906), 68; Collinder I 63; III 88):

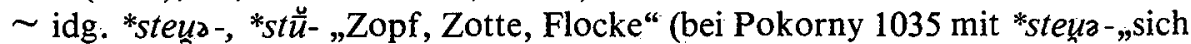
verdichten, sich ballen“ zusammengeworfen) in: ai. prthu-šț́- „mit einem brei-

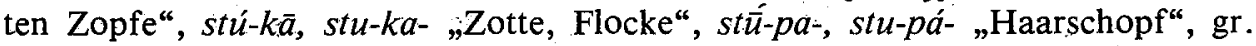
$\sigma \tau U-\pi \pi \eta$, Werg“.

Die idg. Wurzel demnach schon wieder als urspr. *steua $x_{H} H$-anzusetzen und $=$ ural. *tup $(a x) k a !$

Diese zwei: Etymologien sind im Kap. I hinter Nr. (10) nachzutragen.

Inzwischen fand ich weiter, dass die uralischen Affrikatä $c, \breve{c}$ auf indouralisches palatales *t' und zerebrales *t zurückgeführt werden müssen; in in tervokalischer Stellung muss dann als Entsprechung der schwachen (einfachen) ural. Affrikaten im Idg. ein -s-stehen, hinter Guttural dagegen schon wieder - $b$-. Dies suchte ich zu erweisen in Indouralica XIV = Orbis XIX (1970), 282ff. Die Entsprechung im Idg. ist hier also genau dieselbe wie bei reinem $-t$-.

In Pkt. IV werden wir sehen, dass auch andere Möglichkeiten für die Entsprechungen uralischer Laute - $p$ - und $-t$ - auf der idg. Seite in Betracht gezogen wurden.

Niemand jedoch hat es gewagt, an idg. - u- und -s-bzw. - b- zu denken, mit einer einzigen Ausnahme:

Illič-Svityč $227, \mathrm{Nr} .80$ verbindet unser nostrat. *gäti idg. *g'hes- „Hand“, dravid. kac- ds. und ural. *käte ds.,? noch semit.-chamit. *kç ds., jedoch sagt er dazu: „Neobyčnoje sootnošenije i.-e. ${ }^{*} s$, drav. ${ }^{*} c \sim$ ural. ${ }^{*} t$ objasnjajetsja iz ishodnogo * $t i$ (na takoj auslaut ukazyvajet ural.): v poziciji pered $*_{i}$ v nostr. byl, po-vidimomu, predstavlen siljno smjagčennyj allofon ${ }^{*} t$, otražennyj čeredovanijem $S / T$ kak v ${ }^{*} t i$ 'ty' (Sl. č. II). “ Sonst hat er nämlich an der früheren Entsprechung ural. $-t-=$ idg. $-t / d$-festgehalten, s. Pkt. IV. Für uns handelt es sich bei ural. $-t-=$ idg. $-s-/-b$ - u m regelrechte Entsprechungen. 
II. Die wichtigste Frage in betreff der Entsprechungen ural. $-p-=$ idg. $-\underline{x}$ - und ural. $-t-=$ idg. $-s$ - ist, wer hier das Ursprünglichere bewahrt hat, das Uralische oder das Indogermanische.

Auf diese Frage kann man auf zwei Arten antworten: Erstens so, dass man für inlautendes $-p$ - bzw. $-t$ - und ihre idg. Entsprechungen nachzuweisen versucht, wie sich diese Laute im eventuellen Wortanlaut betragen; es ist klar, dass solcher Nachweis nur für einige äusserst günstige Fälle, vor allem mit Suffixen, möglich ist.

So haben wir im Kapitel I (Wortgleichungen) eine Gleichung mit ural. - $p$ - und $-u$ - gefunden, wo das uralische - $p$ - sicher den Anlaut eines sonst selbständigen Wortes ${ }^{*} p \bar{u}$ „Baum? (s. Nr. 11) darstellt; wenn das im Idg. entsprechende - $u$ - sicher auch aus dem Anlaut des selbständigen Wortes für „Baum“ in den Inlaut geraten ist, so muss es einem ural. anlautenden $p$-Laut genetisch gleich sein, also auch selbst einst ${ }^{*} p$ gewesen sein. Denn es ist kaum vorstellbar, dass im Ural. das anlautende ${ }^{*} p$ - in ${ }^{*} p \bar{u}$ durch den Inlaut der Komposita beeinflusst wäre, wo übrigens ebenfalls nur ${ }^{*} p$ steht; ein Wandel von etwaigem urspr. ${ }^{*}-w$ - (das zunächst dem idg. - u- entspräche) zu ural. * $p$ - beim Übergang aus dem Inlaut der Komposita in den Inlaut des selbständigen Wortes ${ }^{*} p \bar{u}$ ist ganz unsinnig, da im ural. Anlaut auch $w$-als eigenes Phonem steht.

Noch beweiskräftiger ist der Fall mit dem Suffix der 2. P. Sg., ural. -t-, idg. - $s-$, s. Kapitel II (Suffixgleichungen) Nr. (5). Hier hat man erstens im Idg. im Pl. die Endung -te (vgl. gr. $\varphi \varepsilon \rho \varepsilon-\tau \varepsilon$, sl. bere-te und ai. Ipf. á-bhara-ta, Brugmann, Grdr. ${ }^{2}$ II 3/2,623ff.). Ähnliches in 2. Sg. Med. (*-thës, Brugmann 645ff.) und Pf. Akt. (*-tha, Brugmann 604ff.), in 2. Du. Akt. (primär *-thes oder *-tes, Brugmann 639ff.; sekundär *-tom ebd. 640ff.) und Med. (ar. *-ithai, *-thai primär, *ithām, -thă̈m sekundär, Brugmann 656f.), so dass der tonolose Verschlusslaut (*-t-bzw. *-th-) direkt bezeugt ist; die isomorphische Opposition idg. $-s-:-t(h)$ - wird sich hier nach meiner Erklärung in Die indoural. Sprachverwandt. und die idg. Laryngaltheorie (Acad. Sc. et Art. Slov. VII/5, 1970, S. 211 Mitte) begreifen lassen: das $-t(h)$ wurde als Verschlusslaut erhalten, wenn unmittelbar davor ein $-H$ - ( = ural. Präsensstammsuffix $-k$-) stand, so etwa *bhérete aus älterem -eH-teH $=$ ural. *-k-te-k; das $-s$ - dieser Endung konnte demnach nur unmittelbar hinter Vokal (= Auslaut des einstigen zweisilbigen Primärstammes oder des mehr als zweisilbigen abgeleiteten Stammes) entstehen, so etwa in Ipf. ai. á-stau-ș aus idg. *é-stēu-s aus älterem *stéue-s (Stamm urspr. zweisilbig: *steue-). Durch isomorphische Analogie kam es dann zur Ausgleichung (im Sg. Akt. ausserhalb der hi-Konjungtion und des Perfekts zugunsten des $-s$, sonst zugunsten des Verschlusslautes). Da es kaum zu glauben ist, dass das $-s$ dieser Endungen ursprüngliche Lautung gewesen wäre und dass erst aus diesem $-s$ das $-t$ - bzw. $-t h$ - der funktionell verwandten Endungen, etwa durch die Wirkung des unmittelbar davorstehenden $H$-Lautes, entstanden wäre, muss man in beiden Lauten, in $-s$ - sowie in $-t(h)$ - zwei kombinatorische Varianten eines einst einheitlichen Phonems sehen. Nun sind die Personalendungen der 2. P. Sg., Du. und Pl. sicherlich ebensowohl auf uralischer wie auf indogermanischer Seite mit dem Stamm des Personalpronomes für 2. P. genetisch identisch; dieser lautet aber klar überall mit ${ }^{*} t$ - an: 
ural. Sg. *ti-: finn. si-nä, lapp. do-n, mordw. to-n, tscher. ta-n, wotj. to-n, syrj. te, ung. të; sam. tawgy ta-nnaך usw., jukagir. te-t (Collinder I 57);

ural. Pl. *te: finn. te, lapp. di, mordw. E $t^{\prime} i-n$ usw., tscher. $t \ddot{a}, t e$, wotj. syrj. $t i$, ung. $t i$; sam. tawgy $t \bar{e}-\eta$ usw.; jukagir. $t i-t$ (Collinder I 62);

idg. (nur Sg.!) Nom. *tü, Gen. *te-une, Dat. *to-i und *te-bhei usw., s. Pokorny 1097; Brugmann, Grdr. ${ }^{2}$ II 2, 383 usw.

Da die selbständige Anwendung dieses Pronomen sicher das Ursprüngliche ist, ist also das $-s$ der 2 . Sg. notwendigerweise als aus älterem $-t$ - entstanden anzusehen.

Es ist auch sonst im allgemeinen anzunehmen, dass der Wandel von $-s-\mathrm{zu}-t$-(also im Falle, das die idg. Lautungen älter wären als die uralischen) viel weniger wahrscheinlich ist als umgekehrt. Ich verweise noch auf den Pkt. V.

III. Die zweite Methode, hinter die Vorgeschichte der interdialektalen Opposition ural. - $p$-: idg. $-u$ - und ural. $-t-:$ idg. $-s$ - und $-\mathrm{p}$ - zu gelangen, besteht darin, dass man ausserhalb des Idouralischen verwandte Sprachstämme aufsucht, die entsprechende Wortstämme und Morpheme besitzen. Ich beschränke mich hier auf das Altaische und zitiere das Material - nur Wortgleichungen - nach Räsänen:

a) ural. $-p-=$ alt. $-p-$ :

finn. ilves „Luchs“ türk. jilpis „Schneeleopard“ usw. (R. 14; sonst im Alt. -b-, wozu Poppe I 85, 87); dazu idg. *lu- „Luchs“, s. Nr. I (4);

finn. korpi „Bruchwald, Bruch, Wildnis; Wüste“ mong. korbi „enges, langes Tal, Vertiefung in der Erde" (R. 33, zum Lautl. s. oben);

finn. kepeä „leicht“ usw. türk. kepäk, keväk „Spreu, Kleie“ (R. 36); vgl. Wortgleichungen $\mathrm{Nr}$. (5);

finn. tapaa "treffen, finden" usw. türk. tap "finden, greifen" usw. (R. 46);

b) ural. $-t-=$ alt. $-t-$ :

finn. kota „Zelt" usw. türk. kota „Haus, Zimmer“, mong. kota „Zaun; alle Haustiere, die in einem Zaune vereingt werden; Stadt, Stadtmauer“, kor. kot „place, locality, site" (R. 5); vgl. auch idg. *kpe-i oben I (29); beachte dort auch lat. casa;

finn. it $\ddot{a}$ - „,keimen“, itä „Osten“ usw. türk. it „stossen“, tschuw. jżt „heben“ (R. 43); Gleichsetzung mit *es- gedeihen oben Nr. (22);

finn. kiitä- „eilen“ türk. kit "gehen“ (R. 44).

$\mathrm{Da}$ auch beim Guttural dieselbe Entsprechung herauskommt, also ural. $-k-=$ alt. $-k$-, z.B.:

finn. oka, oas „Stachel, Dorn“ türk. ok „Pfeil“, mong. oki “Spitze“ (R. 9), muss man zugeben, dass im allgemeinen die altaischen Lautungen genau denjenigen vom Uralischen entsprechen. Doch begegnet man auch andersartigen Fällen, die erkennen lassen, dass auch hier eine Tendenz zum Tönendwerden auf der altaischen Seite am Werke war:

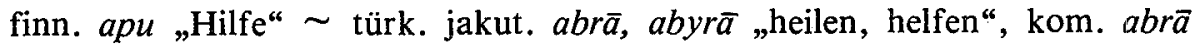
„schützen“, tschuw. opra „schützen, schirmer", mong. abu-ra- „retten, beschützen“ bei R. 35; vgl. idg. *au- Nr. I (1).

Rechnen wir solche Fälle ab, so bleibt sicher, dass auch das Altaische mit Tenuis entspricht, wenn das Uralische in intervokalischer Stellung schwache (einfache, kurze) Tenuis besitzt. 
Es ist demnach wenig wahrscheinlich, dass die idg. spirantische Aussprache das Ursprüngliche darstellen würde.

IV. Hier muss ich die Behandlung unserer zwei Entsprechungsgesetze, wie sie unter Pkt. I dargestellt wurden, auf kurze Zeit verlassen, um ein anderes En tsprechungsgeset $z$ zu besprechen, das lange vor mir schon andere aufgestellt haben, und zwar:

ural. einfaches $-t-=$ idg. tönendes $-d$ -

Dies Gesetz ist durch folgende Gleichungen belegt:

(1) finn.-ugr. *pata „Topf“ in finn. pata ds., tscher. pat, pot „eiserner Topf, Kessel (von Kupfer od. Eisen)“, wog. pōt, püt „Topf“, ostj. put „Kochtopf, Kessel“, ung. fazék "Topf", sam. unsicher (Collinder I 47):

$\sim$ idg. *pă/őd- „Gefäss, Topf“ in ahd. fazz „Behälter, Kasten“, asächs. fat Ntr. „Gefäss“, ags. fầt ds., „Fass“, anord. fat „Gefäss, Kleid“, ags. fäetels „Gefäss", ahd. givazzi „Bagage“, mhd. gevaezze, nhd. Gefäss, lit. púodas, lett. puôds "Topf“.

Pokorny 790 fasst alle diese Wörter unter *pё d- „fassen“ und „Gefäss“ zusammen, doch ist diese Wurzel in verbaler Funktion m. E. zur folgenden Nr. zu stellen; über ai. palla- M. „Kornbehälter“, palli „Getreidemass“, die eventuell auf *pad-lzurückgehen, s. Mayrhofer, Altind. EW. II 236 (sehr unsicher).

Die Etymologie, die Collinder, IU. 67 (*pata zu ags. pott „,irdener Topf“) nach älteren zitiert, ist schon wegen des Vokalismus kaum annehmbar.

(2) finn.-ugr. *pitä- „halten, heften“ in finn. pitä- „halten“, mordw. E ped'a-, M ṕed’ - „sich anschliessen, kleben“, „anhaften“, „eigensinnig anfangen“, tscher.

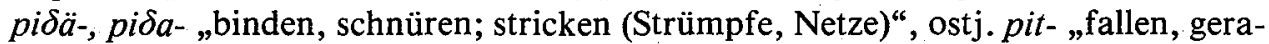
ten; anfangen, werden“, ung. füz „schnüren; fädeln; reihen; flechten; knüpfen, heften“, füzér „Kranz, Schnur, Girlande“ (Collinder I 108; Steinitz, Gesch. des finn.ugr. Vok. 60f., Nr. 333 mit Weiterem):

$\sim$ idg. *p"̄e $d$ - „fassen“ in ahd. fezzil, mhd. vessel „Band, Fessel“, ags. fetel(s) „Gürtel“, anord. fetill „Schulterband" aus urgerm. *fatila-z, anord. fäeta "es mit jem. zu tun haben“, lit. pëdas „Getreidegarbe“, lett. pêda „Bund, Armvoll“.

$\mathrm{Zu}$ dieser Sippe Pokorny 790, wo jedoch wohl fälschlich die Sippe für „Fass, Gefäss" eingeflochten wird. Es wird sich um zwei genetisch verschiedene, urspr. im Vokal der ersten sowie der zweiten Silbe unterschiedliche Sippen handeln, die auf Grund einer lautlichen und bedeutungsgeschichtlichen Konvergenz später nicht mehr rein auseindergehalten werden konnten, vgl. vor allem ahd. fazzōn, mhd. vazzen ,in ein Gefäss tun“ von Fass, dann „fassen, rüsten, sich bekleiden“, s. KlugeMitzka, Deutsch. EW. ${ }^{20} 186$ s.v. S. noch oben Nr. (27) (pitä-).

(3) wichtig ur. wete „Wasser“ in finn. vesi vete-, "mordw. ved”, tscher. $\beta_{\gamma}$ t, $\beta \ddot{u} t$, wotj. vu, syrj. va, wog. $\beta i t$, ung. viz $\sim$ vize-; sam. jur. jì' jizd-, waldjur. $\beta$ 'it, tawgy. be' $\sim$ beda- usw. (Collinder I 67):

idg. *aued- „benetzen, befeuchten" und „Wasser“ (Pokorny 78-80) z.b. verbal in ai. ódatī „die Quellende, Wallende“, $u$-ná-d- „quellen, benetzen“; im Wort

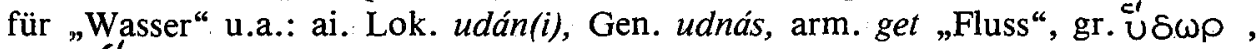

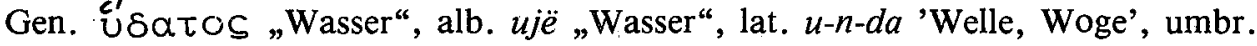


utur 'Wasser', Abl. une, got. watō „Wasser“, anord. vatn, vatr, ahd. wazzar, asächs. watar, ags. waeter, lit. vanduõ, unduõ, lett. ûdens „Wasser“, aksl. voda, heth. uatar, Gen. unetenaš ds.

Alte Gleichung, vgl. Collinder, IU. 70f.; U. 126.

Misslich ist a) dass die idg. Wurzel im Anlaut ein weiteres * $a$-besitzt, das auf eine kürzere Wurzel *au-zurückzuführen lässt (s. bei Pokorny), b) dass die Wurzel im Idg. ursprünglich verbal ist. Der erstere Einwand ist recht ernst, denn die kürzere Wurzel idg. *au- geht nach meinem Vorschlag in UAJb. 44 (1972), 165 (ich habe übersehen, dass schon vor mir Skalička, UAJb. 41 (1969), 337 dieselbe Etymologie vorgeschlagen hatte) zu finn.-ugr. *uwa „Strom" in finn. vuo usw. Wir müssen demnach in ural. *wete eine deverbale Ableitung sehen (ähnlich Skalička a.a.o.), die im Falle der Identität mit idg. *aued- ursprünglich dreisilbig war (*uwe-te), was ganz wahrscheinlich ist (zum Abfall des anlautenden Vokals, der ohnehin noch homorgan ist mit folgendem *-w-, vgl. ural. *wü-lä, *wü-le ,oben, oberer, das Obere“ aus * $\ddot{u} w e-l \ddot{a} / e$ in UAJb. $44,165 \mathrm{f}$.); auf beiden Seiten wurde dann ein genetisch identisches Suffix ural. ${ }^{*}-t e-=$ idg. ${ }^{*}-d$ - benutzt (andere idg. Vertretung dieses Suffixes oben Kapitel II Nr. (6): -s-, hier regelrecht).

Auch die verbale Funktion von idg. *aue-d-kann sich als eine Folge alter idg. Tendenz zur Verbalisierung der Nominalstämme begreifen.

Wenn also die oben gegebene Analyse richtig ist, dann ist diese Gleichung trotz der idg. Vertretung $-d$ - des ural. $-t$ - als ein festes Bauelement in unser indouralisches Gebäude einzufügen.

(4) ural. *wet $\ddot{a}$ - „ziehen, führen“ in finn. vet $\ddot{a}$ - ziehen, zerren, schleppen“, mordw. E véd'a, vit'i-, $\mathrm{M}$ v́ed'a-, auch veto- „führen, leiten, bringen“, tscher. $\beta \ddot{u ̈ \delta e-, ~} \beta \mathrm{i} \delta e$ - „leiten, führen“, ung. veze-t- „führen, leiten, lenken, geleiten“, vezér „Führer"; unsicheres im Sam. (Collinder I 67):

$\sim$ idg. *ued $(h)$ - (nach Pokorny *uedh-, vor Nasalen *ued-) „führen; heimführen, heiraten (vom Manne)“, mit - $d$-in gr. Ésvov „Brautgabe“, ags. weotuma, wituma, wetma M. „Kaufpreis der Braut“, afries. wetma, witma ds., burg. wittemo; dazu m.E. auch toch. B $w \bar{a} y-$-, A $w \bar{a}-$, Suppletivstamm zu $\bar{a} k$ - „führen“, sicherlich aus einer $d$-Form unserer Wurzel, mehr (mit Lit.) an anderer Stelle; - mit ${ }^{*}$-dh- ai.

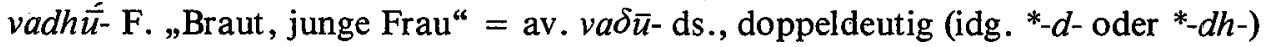
av. $v \bar{a} \delta a y a-$ "führen, ziehen“, air. fedid „führt, geht, trägt, bringt" usw., lit. vedì, vèsti „leiten, führen; heiraten (vom Manne)“, aksl. vedo, vesti „führen“ usw.

Alte Gleichung, s. Collinder, IU. 71; U. 126 (wo Entlehnung aus dem Indogermanischen als nicht ausgeschlossen betrachtet wird).

Das idg. *-dh-muss jetzt, da das Toch. ein ${ }^{*}$ - $d$ - ausserhalb nasaler Nachbarschaft befürwortet, als aus ${ }^{*}-d$ - + Laryngal $-H$ - zussamengesetzt gelten, so kommt sofort die Gleichung ural. $-t-=$ idg. $-d$-heraus.

(5) der Stamm finn.-ugr. *käte „Hand“ wurde oben I Nr. (26) mit idg. *g'hes ds. identifiziert; doch kann man auch an idg. *ghed- „fassen, anfassen, ergreifen" bei Pokorny 437f. denken, das in etwa folgenden Wörtern fortlebt: gr. xavsavw, Aor: દ̌xaסov usw. „fassen“, alb. gjëndem „werde gefunden“, gjënj usw. "finde“, lat. 
prae-hendō „fassen, anfassen, ergreifen“, praeda „Beute“ $<$ *prai-hedā, air. ro-

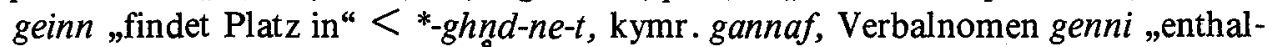
ten sein, Platz haben“, got. bi-gitan, "finden „, anord. geta „erreichen; hervorbringen, vermuten“, ags. for-gietan "vergessen“, ahd. pi-gezzan "erlangen ", fir-gezzan "vergessen“.

Die idg. Wurzel ist ursprünglich nasallos, das $-n$ - ist Präsensinfix; demzufolge ist es unrichtig, dies *ghe(n)d-mit einem finn.-ugr. *kunta- „anfassen usw.“ (die Sippe bei Collinder I 28) zusammenzustellen, wie Collinder, IU. 61f. und U. 123 tut; die richtige Entsprechung des ural. ${ }^{*} n t$ - ist idg. $-n d h$ - oder $-d h-$, wie ich in Orbis XXII (1973), 5ff. gezeigt habe.

Jedoch ist die dreifache Gleichung finn.-ugr. *käte „Hand" $\sim$ idg. *ǵhes ds. *ghed- „fassen, nur unter folgenden Voraussetzungen gutzuheissen: a) die Verbalisierung der Wurzel im Idg. ist auch sonst oft anzutreffen, vgl. schon hier oben Nr. (3); aus „mit der Hand hantieren“ entstand ganz leicht ein „fassen, greifen“; b) idg. *ghed-hat anlautenden Velar, wenn man den alb. Zeugen glauben darf; doch begegnet man im Alb. nicht selten auch sog. westindogermanischen Elementen, die statt des zu erwartenden Palatals einen Velar aufweisen, so z.B. alb. quhem "heisse" aus idg. *klu-sk-. Man kann also annehmen, dass alb. gjënj, gjëndem aus dem Illyrischen = einer Kentumsprache stammt, was bei der bekannten Vielgestaltigkeit des Albanischen gut verständlich ist.

Die Ratio der Spaltung in *ghes und * g'hed- wird natürlich unten am Ende dieses Pkt. zur Sprache kommen.

(6) Die regelrechte Entsprechung des ural. Ablativ-Partitiv-Suffixes -ta ist idg. $-s$, s. oben Kapitel II Nr. (4). Doch verglich man bisher eher das idg. a blativische $-t / d$, das in folgenden Kasusformen zum Vorschein kommt:

a) im regelrechten Ablativ Sg. der nominalen und pronominalen $o$-Stämme: ai. vr̆kāt „vom Wolfe“, av. vahrkät ds., gr. delph. Fol $\mathcal{H} \omega$ "domo“, alat. Gnaivōd, got. hwaprō "woher", lit. vilko = sl. v6lka „des Wolfes“ (s. Brugmann, Grdr. ${ }^{2}$ II 2, 165); ai. tásmāt usw. „von dem“ usw. (Brugmann 362); Adverbien: z.B. *t ${ }^{*} d$,von da an, dann“ im Imperativ ai. bhára-tāt $=$ gr. $\varphi \varepsilon \rho \varepsilon-\tau \omega$, lat. fer-tōo(d); ai. tất , ,infolge davon", kret. $\tau \widetilde{\omega}-\delta \varepsilon$,hinc“, aksl. ta-že „und dann, itaque“ (Brugmann 697) usw.; hier also immer mit Dehnung des Stammauslautes und Zirkumflex;

b) im Ablativ Sg. der Personalpronomina und Verwandtes: ai. má-t "von mir“, $t v a ́-t$,von dir“, av. ma-t, ä $t$-, apers. $\breve{s} a^{h}$ „von ihm“, lat. $m \bar{e}(d), t \bar{e}(d), s \bar{e}(d)$; auch im Dual und Plural: ai. asmá-t, yuşmá-t = av. ahma-t, yušma-t „von uns, euch“, ai. $\bar{a} v a ́-t$, $y u v a ́-t$,von uns, euch beiden“; gr. in den Zusammensetzungen: $\grave{n} \mu \varepsilon-\delta-\alpha \pi \delta \varsigma$, der unsere, einheimisch“, vgl. ai. asmad-íya- „unser“ und Frisk, Gr. EW. I 635; $\alpha \lambda \lambda \mathrm{\alpha}-\delta-\alpha \pi \delta$ s,von anderswoher, fremd“ usw., s. Frisk 76.

Die durchgehende Länge und schleifende Intonation in der Gruppe a), fast durchgehende Kürze (auch kann hier die Länge analogisch nach a) eingeführt sein) in der Gruppe b) zeigen deutlich, dass die Gruppen nicht beide auf dasselbe Formans zurückgreifen können: in der Gruppe a) muss es einst einige prosodische Faktoren gegeben haben, die den quantitativen und tonematischen Unterschied gegenüber b) zur Folge hatten, d.h. die Gruppe a) muss neben allgemein gültigem Ablativ- 
Partitiv-Suffix indoural. *-ta vor diesem noch etwas anderes enthalten haben; dies war ein zusätzliches Affix (Koaffix) $-k$-, auf das ich in meiner Abhandlung Die indoural. Sprachverwandt. und die idg. Laryngaltheorie (Acad. Sc., usw. VII/5), S. 213 ( $=$ S. 31 des Sep.) zu sprechen kam: indoural. Ablativsuffix ${ }^{*}-k$-ta $=$ idg. ${ }^{*}-H-t$ : hier hat das vorausgehende $-H$ - das $-t$ vor der Assibilierung bewahrt.

Anders steht es mit der Gruppe b): hier ist der Vokal vor dem - $d$ ursprünglich kurz, das Ablativ-Suffix muss hier in seiner einfachsten Form indoural. *-ta vorgekommen sein, man hat also das Recht, hier mit der Entsprechung ural. $-t-=$ idg. $-d-$ zu rechnen.

Diese schon alte Gleichung ist also nur bedingt richtig, trotz Collinder, IU. 21f., U. 131.

(7) Collinder, IU. 22ff. bespricht eine spezielle Kas us -Rekt u s-Bildung mittels eines ural. $t$-Suffixes, die besonders gut im Lappischen zum Vorschein kommt: lapp. *-ta z.B. in N gu-tte, Gen. gu-de „welcher" (Interrog.), L $k u$-כti „welcher“ usw.; $\mathrm{N} d \bar{a}-t$, Gen. $d \bar{a}-m$ "dieser hier, dieser“, $d u o-t$ (Gen. $d u o-m$ ) „der dort (weiter weg), jener" usw.;

tscher. $k u-\delta \bar{\partial}$,wer, welcher; irgendeiner";

tscher. $m a-\delta a$ „was“, wog. $m \ddot{a}$-tä „,irgend welcher“, ostj. $m a-t$ „welcher“;

auch sam.

Vgl. noch Lehtisalo 390ff.

Collinder a.a.O. verbindet dies uralische Suffix mit dem idg. pronominalen $-d$ des Nom.-Akk. Ntr. Sg.:

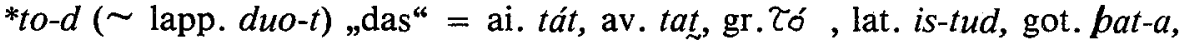
ahd. daz, asächs. that;

*qu $u_{o-d}$ "welches" $=$ ai. kát, av. kat, lat. quod, osk. púd, ahd. hwaz; heth. kuuat „warum“; usw.

Vgl. Brugmann, Grdr. ${ }^{2}$ II 2, 358.

Falls die morphologischen Voraussetzungen, die Collinder a.a.O. an den Tag bringt, stichhaltig sind, kann man erneut von einer Ensprechung ural. $-t-=$ idg. $-d-$ sprechen. Ich muss Collinder unbedingt beistimmen, obwohl das Verhältnis ural. Suffix ${ }^{*}-t a \sim$ idg. ${ }^{*}-d$ systemgeschichtlich auch ein wenig anders aufgefasst werden kann.

In Orbis XIX (1970), 282ff. habe ich die Entsprechungen uralischer inlauten-

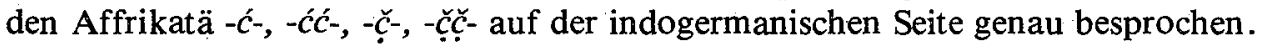
In einigen Fällen scheint die Entsprechung ural. $-\check{c} k-=$ idg. $-d$ - (urspr. ${ }^{*}-d H$-?) herauszukommen, die eigentlich auf der ural. Seite ein starkes, gedoppeltes *-çç- vor $k$ - verlangt; vgl. a.a.O. $\mathrm{Nr}$. (19) und (20), wogegen regelrecht $\mathrm{Nr}$. (14)-(18). Wenn in den Nr. (19) und (20) wirklich alte einfache Affrikata steckt, so muss man das idg. $-d$ - als unregelmässige Entsprechung ansehen, die in den Rahmen der hier besprochenen mit ural. $-t-=$ idg. $-d$ - gehört; denn a.a.O. S. 315 habe ich festgestellt, dass die uralischen Affrikaten aus einstigen palatalisierten und zerebralisierten dentalen Verschlusslauten hervorgegangen sind: ural. $-\dot{c}-<$ indoural. $* * t$ ', ural. $\breve{c}<$ indoural. ${ }^{* *} t$ usw. So kann man die eben genannten Nr. (19) und (20) auch hier einfügen. Und dazu kommen noch folgende neue Gleichungen, ebenfalls mit der Entsprechung ural. Affrikata: idg. $-d-$; die ural. Affrikata war schwach (einfach, kurz): 
(8) finn.-ugr. *keç̆ne „Wacholder, Iuniperus communis“ in lapp. gâskâs, S gâs†ěsě, syrj. kaç-pomel' (pomol', pomel', auch ponel', ,junge Fichte, junger, kleiner

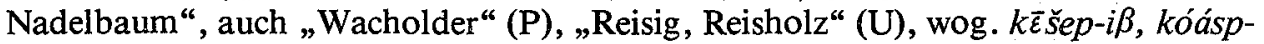
ji $\beta$ (ji $\beta$ „Baum“) (Collinder I 79; Toivonen, FUF. XIX (1928), 134, Nr. 202; LytkinGuljajev 118 usw.):

$\sim$ lit. kadagỹs „Wacholder“, lett. kadags, kadęgs, apreuss. kadegis, wahrscheinlich noch gr. $\mathcal{H} \dot{\delta} \delta \mathrm{S}_{\mathrm{S}}$ "Wacholder", später „Zeder“; über die Schwierigkeiten bei der Verbindung dieser idg. Wörter s. bei Frisk, Gr. EW. I 808; Weiteres, auch zur Frage von der Entlehnung des finn.-ugr. Wortes aus dem Balt. und umgekehrt, s. Fraenkel, Lit. EW. 202.

Ich betrachte als gemeinsam die Wurzel ural. *keç- = idg. *qed-bzw. (balt.) *qod-; das Übrige sind Suffixe, im Wog. ein $p$-Formans, im Gr. ein ro-Formans, schliesslich ein $\eta$-Formans im Lapp., mit welchem ich das balt. - $g$-identifiziere (zur Entsprechung ural. - $\eta$ - = idg. - g- s. KZ. 84 (1970), 151ff. = Indouralica VII). Man steht also vor der recht bestechenden Möglichkeit, finn.-ugr. *keçॅe (aus dreisilbi-

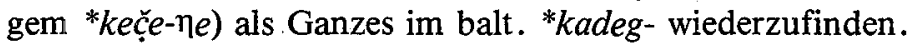

Das idg. *ged- „rauchen, russen“ bei Pokorny 537 betrachte ich als userer Sippe fremd; zur uralischen Verwandtschaft vgl. Verf., Orbis XIX (1970), 296 Nr. (18): finn.-ugr. *küçç̌e- oder *küçe- (dann zu unseren hier besprochenen Fällen) „verdeckt brennen, glimmen“.

(9) ural. *puçę- „fallen“ in finn. putu „Abfall, Schutt, Kehricht" putoa-, Inf. pudota „Von einer Höhe fallen; entfallen“, pudottaa „fallen lassen, fällen“, pudista„schütteln, ausschütteln, rütteln“, estn. pudene-, pudise-, pudu- „zerfallen, sich zerbröckeln, abfallen“, pudise- auch „beben“, mordw. E počt, M počf „Mehl“, E počaa, $M$ počana „locker, krümelig“, počaksa- „schmeissen, ausseinanderschmeissen, zerstreun“, E počodo- „streuen, bestreuen, schütten“, M počara-ds., tscher. KB poča ฤgausw. „sich werfen, sich wälzen“, syrj. buždy- „umfallen, einfallen, einstürzen, verschüttet, weggespült werden“, ostj. pōç̆ðyin- „fallen, zu Boden stürzen“ usw.; Unsicheres im Sam. (Toivonen, FUF. XIX (1928), 84, Nr. 74; Collinder I 53 usw.):

$\sim$ idg. *ped- „fallen“, sekundär "gehen“" bei Pokorny 791 (Weiteres, so *pedM. „Fuss“, *pedo-m „Fussstapfe, Schritt, (Fuss)boden, Platz" ebd. 790ff.) z.B. in

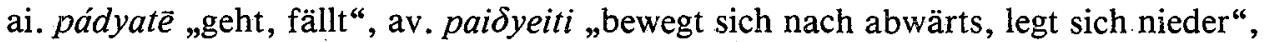

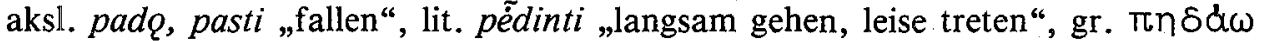
"springe, hüpfe“, lat. Kompar. peior „schlechter“ $<{ }^{*}$ ped-iōs „mehr abfallend“, pessimus < *ped-temo-s „der schlechteste $=$ am meisten abfallende“, pessum „zu Boden, zugrunde“ = ai. Inf. pát-tu-m, anord. feta "den Weg finden“, ags. ge-fetan "fallen“, ahd. fezzan "labare“ usw.

Auch im Idg. ist die Bed. „fallen“, „sich zu Boden bewegen“ klar die ursprünglichste.

Da die schwachen Affrikaten ural. $-\dot{c}$ - und -čc-im Idg. genau so durch $-s$ - wie die schwache dentale Tenuis ural. - $t$ - vertreten werden, wenn es sich um normale, ungestörte Entwicklung handelt, so muss man die Vertretung durch idg. $-d$ - als etwa kombinatorisch, d.h. durch besondere, in unmittelbarer Nähe des fraglichen Lautes liegende Faktoren verursacht erklären. In Betracht kommen m.E. folgende spezielle 
Faktoren bzw. Bedingungen, die am Entstehen des idg. $-d$ - schuld sein können:

a) in unmittelbarer Nachbarschaft des regelwidrigen $-d$-befindet sich sehr oft ein Nasal, und zwar vor $-d$ - oder hinter ihm:

vor allem ist *ghe-n-d "fassen" Nr. (5) zu nennen: lat. prae-hendo samt gr. $x \propto \nu \delta \alpha \nu \omega$, weiter die Formen mit idg. - $n$ - in der Schwundstufe, gr. $\stackrel{x}{\varepsilon} \times \alpha \delta \circ \nu$, air. ro-geinn, kymr. gannaf usw.; gr. Fut. $\times \varepsilon l \sigma o \mu \alpha \iota$ aus *ghend-s-, Pf. $\varepsilon \times 0 \vee \delta a$, alb. gjëndem usw.; alles dies macht wahrscheinlich, dass das ursprünglich infigierte - $n$-später; wenigstens auf gewissen dialektischen Gebieten (im. Gr. und Kelt.), als Wurzelbestandteil aufgefasst wurde, so dass sogar eine Schwundstufe mit $-n$ - entstehen konnte; im Lat. dagegen zeugt praeda noch von der richtigen Auffassung des präsentischen $-n$-;

im Wort für „benetzen“ usw. Nr. (3) kam gleichfalls ein mit - $n$-infigiertes Präsens vor, vgl. ai. unád-, lat. unda, lit. vanduõ und unduõ, lett. ûdens (aus *und-), apreuss. wundan, unds; dazu kommt, dass das Paradigma von "Wasser" in den Ka-

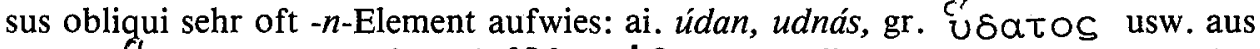

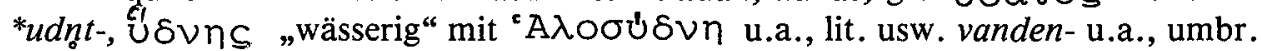
Abl. une aus *udni, got. wato, watn-, anord. vatn, heth. ueten-;

idg. *ued(h)- „führen“ Nr. (4) hat wenigstens im alten Nomen für den „Kaufpreis der Braut" ein nasales Suffix aufzuweisen: gr. $\stackrel{\complement}{\varepsilon} \delta$ vov u.a., ags. weotuma usw.; dazu das Verbalnomen kelt. *ved-no- in mkymr. ar-wein zu ar-weddu „führen, bringen";

der Ablativ auf *-d Nr. (6) kommt wenigstens im Gr. ständig vor einem $n$ Element: $\alpha \lambda \lambda \circ \delta-\alpha \pi \delta s, \tau \eta \lambda \varepsilon \delta-\alpha \pi \delta s, \pi \alpha \nu \tau o \delta-\alpha \pi \delta s, \pi \circ \delta-\alpha \pi \delta s$, $\stackrel{\leftarrow}{n} \mu \varepsilon \delta-\alpha \pi \delta s$, Ú $\mu \varepsilon \delta-\alpha \pi \delta s$ aus idg. *-nqún-s = lat. -inquus;

dazu kommt, dass die Nr. (8) im balt. ${ }^{*} k a d e g-$ ein uraltes (indoural.) $\eta$-Suffix enthält, $\sim$ finn.-ugr. *keç-ne;

schliesslich kommen im Paradigma von *ped- „fallen“ (urspr. athematischer Aorist) oft Formen mit Nasal im Anlaut der Personalendungen. vgl. ai. VS. apadmahi, RV. apadran (für urspr. *é-ped-nto?); auch das sl. pado scheint auf ein älteres athematisches Paradigma zurückzugehen, wie Meillet, MSL. 19 (1916), 183 dargetan hat.

Auch ist er sehr wahrscheinlich, dass in beiden Endungen mit $-d$, im Abl. Nr. (6) und Nom. Akk. Ntr. Nr. (7), oft das Sandhi entscheidend war: z.B. *tód né esti „das ist nicht"; *me-d, *tue-d, *nsmé-d usw. *mìueti „von mir, dir, uns schiebt er weg".

b) es konnte beim werdenden $*_{-}-b$ - z.T. zur dissimilatorischen Ablenkung des Entwicklungsganges in der Richtung zum Verschlussbilden kommen, wenn in der nächsten Silbe (in deren Anlaut) ein weiteres *-b- oder sogar ein reiner Sibilant (voruridg. $-s-,-s-, \breve{s-})$ stand:

so könnte z.B. *top eb- als Vorstufe von *tód ésti „das ist“ zu Nr. (7) zunächst zu *tod eb-dissimiliert worden sein; zu *eb = später *es- s. Kapitel I Nr. (22);

ein *uepep- „Wasser" mit dem Suffix Kapitel II Nr. (6) oder (8) zu *aued-Nr. (3) (ural. * $(u$ )wete) kann zunächst zu *uedeb-dissimiliert worden sein, erst daraus idg. *uedes- „Wasser“, erhalten in ai. utsa- „Quelle, Brunnen“, air. u(i)sce „Wasser" aus *udeskiïo-; 
das idg. *päa/ŏdo- „Fass, Gefäss“ muss ursprünglich ein Wurzelnomen gewesen sein, = finn.-ugr. *pata, demnach urspr. *pap; hier waren z.B. der Gen.-Abl. Sg. mit Endung *- $b$ (s. oben Kapitel II Nr. (4)!) und der Nom.-Obl. Pl. (daraus Lok. Pl.) ebenfalls mit der Endung *-p (s. Kapitel II Nr. (3)!) die mutmasslichen Grundlagen für das Entstehen von $-d-:{ }^{*}$ pape- $b>{ }^{*}$ padep usw.;

das athematische *ped- „fallen" kann sein $-d$ - auch aus der 2. P. Sg. geschöpft haben, wo die Personalendung urspr. mit *-p- anlautete, vgl. Kapitel II Nr. (5): idg. *é-ped-so demnach aus urspr. *pépe- $b$-.

c) auch ein tönender velarer Spirant $* \gamma$ könnte in gewissen Fällen einen vorhergehenden Spiranten $* b$ zum Verschlusslaut $* d$ werden gemacht haben, so in Nr. (4), idg. *ued $(h)$ - „führen“: urspr. wenigstens zum Teil *ueb $\gamma$-(schwere Basis mit konsonantischem Schwa) $>{ }^{*} u e d \gamma-=$ ai. vadh- in vadh $\bar{u}-\mathrm{F}$. „Braut“;

ebensolche Kraft könnte einst auch das vokalische - - - gehabt haben, so dass pa-

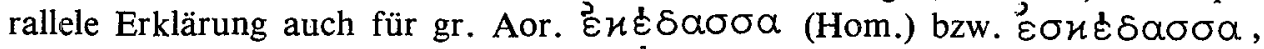
- aoa (ebenfalls Hom.) von $(\sigma) h \varepsilon \delta \& \nu \cup \cup \mu \iota$,zersplittere, zersprenge, zerstreue" möglich wäre: nach Orbis XIX (1970), 297 gehört dies Verbum (zweisilbige Basis) zu finn.-ugr. *kaç̌ke- (also mit Guttural = idg. -ə-) „entzwei gehen, zerbrechen".

Auch mit tonlosem Spirant $-H$ - könnte man in beiden Fällen operieren.

Die drei Faktoren waren, wie ersichtlich, von sehr verschiedenem Charakter, so dass man das Entstehen von - $d$ - auf ganz verschiedenen Wegen vor sich gehen lassen muss:

a) eine echte Dissimilation ist bei zwei aufeinanderfolgenden $-\mathrm{b}-(\mathrm{Pkt}$. b) anzunehmen;

b) ein Tönenwerden muss für die Fälle unter a) angenommen werden: beim vorhergehenden oder nachfolgenden $-n-,-m-,-\eta-;$ dass ein unmittelbar vorausgehendes $-n$ - das $-p$ - in' eine Stufe - $d \delta$ - dränge, s. Orbis XXII (1973), 5ff.;

c) auch vor „Laryngal“ $(H, \gamma)$, s. Punkt c), musste es zur Dissimilation kommen (zwei Spiranten aufeinander!).

Beim Dissimilationsprozess wählte die Sprache wohl deswegen das $-d$ - als Endprodukt, weil das - $t$ - als Tenuis wohl Fortis war, demnach mit lockererem -p- kaum genug verwandt. In der Nähe des Nasals degegen kam es wohl zu einem Kompromiss zwischen tönendem $-\delta$-vor Nasal und einem Doppellaut $-d \delta$-nach Nasal; dieser Kompromiss ist also Folge der Tendenz zu einem Ausgleich im selben Paradigma.

Auf der Grundlage dieser kombinatorischen Variationen kam es folglich zu einem Wechsel idg. - $s$ - (regelrecht): $-d$ - (kombinatorisch). Zum Teil spaltete sich einheitliche etymologische Gruppe in zwei selbständige Einheiten, so sicher idg. *'ghes„Hand“ Kapitel I Nr. (26) gegenüber idg. *ghed- (mit. *gh-?) oben Nr. (5); zu beachten ist, dass im Paradigma des Wortes für „Hand“ kein Faktor besteht, der zu $-d$ - führen könnte.

Auch zwischen gen.-abl. idg. -s Kapitel II Nr. (4) und dem rein abl. - $d$ oben Nr. (6) bestand einst ein isomorphisches Wechselverhältnis, doch muss dies bald aufgehört haben.

Hier ist es am Platze, noch ein Beispiel solchen Wechsels vorzuführen, das bisher noch nicht zur Sprache kam: 
$\mathrm{Zu}$ ural. *wete „Wasser" (urspr. *uwe-te?) stellten wir oben unter Nr. (3) das idg. verbale und nominale *aued-. Daneben besteht aber auch ein idg. *ues- „feuchten, nass" bei Pokorny 1171f., z.B. ahd. wasal "Regen", ags. wōs "Feuchtigkeit, Saft“, älter dän. norw. $\bar{o} s$ „Pflanzensaft“ u.a. Im allgemeinen ist hier die Lage so, dass kein $n$-Element an diese Wurzel tritt (ausser in ahd. waso „Rasen, Erdscholle, Grube", nhd. Wasen „Rasen“, wenn. hierher). In diesem *ues- steckt also die regelrechte Vertretung des indoural. *uwe-te. Natürlich schwand aber das Bewusstsein eines etymologischen (urspr. auf paradigmatischem Wechsel $-s^{-}:-d$ - fussenden) Zusammenhanges zwischen idg. *aued- und *ues-; die beiden Gruppen wurden verselbständigt .

Trotz der endgültigen Spaltungen einst zusammenhängender Systeme mit Wechsel $-s-:-d$, die oben beobachtet wurden, kann man aber vermuten, dass solcher Wechsel einst gang und gäbe war. Er bestätigt seinerseits die Ergebnisse, die wir in diesem Aufsatz gemacht haben: spontane Entsprechung des schwachen intervokalischen - $t$ - (und $-\dot{c}-$, $-\breve{c}_{-}$) der uralischen Sprachen ist im Idg. $-s-$, unter speziellen Bedingungen dagegen tritt - $d$ - auf.

V. Doch ist die Lage in Wirklichkeit noch komplizierter. Es wurde nämlich ein Beispiel festgestellt, das im Uralischen schwaches intervokalisches - $t$ - aufweist, auf der idg. Seite dagegen entspricht diesem Konsonant ebenfalls - $t$-. Das ist der folgende Fall:

finn.-ugr. *wote „Jahr-, in finn. vuosi vuote- ds., wotj. vapum, Glazow uapum „Zeit, Lebenszeit“ (pum „Ende“), syrj. vo, $u$ „Jahr“, ostj. al Nom. Sg. + Personalsuff. $\bar{u} l \hat{\partial}-m(\mathrm{~V})$, ot (Pl. $\bar{o} D \hat{\partial} t)$ (DN usw.) „Jahr“, weiter lapp. S -vùöota Gen. -vùöta in nùörra-vùösta „Jugend“ (nùörra „jung“), ung. -val, -valy in ta-val(y) „im Vorjahre, voriges Jahr“ (ta- finn. tuo „der, jener“) (Szinnyei, Nyelvh. ${ }^{7} 37$, 57f., 60; Collinder I 126 usw.):

idg. *uet- „Jahr“ in heth. uitt-ds. (Nom. Sg. uizza in uizza-pant- „alt“, Dat.Lok. yitti usw.), tiefstufig *per-ut „im vergangenen Jahre“ $=$ ai. parút, sonst durch $-i$ verdeutlicht (Lok. Sg.) *per-uti ds. in arm. heru, gr. $\pi \varepsilon \rho \cup \sigma \iota$, dor. $\pi \varepsilon \rho \cup \tau \iota$, anord. fjaro, mhd. vert ds., air. ónn-urid „ab anno priore“; dazu viele Ableitungen mit Suffix - (e)s-, die oben im Kapitel II Nr. (8) aufgezählt wurden; konsonantischer Stamm ohne Suffix noch im gr. ( $\varepsilon \grave{\zeta})$ v $\varepsilon \omega \tau \alpha$,aufs neue (nächste) Jahr“, wohl. aus *neue-uat- $a$ mit altem - $a$ - $\sim$ finn.-ugr. - $\bar{o}$ - (vgl. zu anderen Erklärungen Frisk, Gr. EW. II 312). Idg. Material bei Pokorny 1175.

Diese Gleichung ist alt, vgl. Collinder, IU. 72 nach Schrader; doch nicht mehr in U. - Der Konsonantismus - der innere Dental - im Finnisch-Ugrischen ist nämlich recht schwierig: das Ostj. und das Ung. sprechen für urspr. *- $\delta$ - und so hat Collinder III 109 unser Wort zu den $\delta$-Wörtern gestellt und S. 414 als Urform *o $\delta e$ aufgestellt. Im Permischen dagegen ist die Normalvertretung von $-\delta$ - ein $-l$, das nur unter besonderen Bedingungen schwindet, so dass man auf Grund das Perm. eher an *wõte denkt, mit anlautendem *w-; da das Ostj. auch hinsichtlich des anlautenden Halbvokals (spurlos verschwunden?) abweicht, scheint es mir geraten, das ostj. ${ }^{*}$ ola $x$ (aus ${ }^{*} o \delta a^{x}$ ?) fernzuhalten. Szinnyei 37 zitiert nun unsere Sippe unter den $t$-Wörtern, jedoch als besondere Gruppe, wo das finn .-ugr. - $t$ - im Ung. durch - $l$-(aus der Schwachstufe $-\delta$-) vertreten wird; so noch in der 2. Sg. $-l$ (Szinnyei 121) und im 
Abl. auf -l (Szinnyei 131f.). Wenn auch Widersprüche möglich sind, scheint es mir doch geraten, auch -val(y) als zu einer Art Formans herabgesunken auf dieselbe Weise zu erklären.

Es ist wohl erfreulich, dass Lytkin-Guljajev 59 zur Urform mit - $t$ - wiederkehren, obwohl auch $*_{w}$ - im Anlaut wieder zur Ehre zu bringen wäre. Jedenfalls steht es jetzt fest, dass finn.-ugr. ${ }^{*}$ wote $=\mathrm{idg} .{ }^{*}$ uet- ist, mit unregelmässiger Vertretung des urspr. - $t$ - im Idg.

Ich habe oben Kapitel II Nr. (8) luw, ušša- = hgl. usa- „Jahr“ bei meiner alten Erklärung aus *uet-s-o- = ai. vatsá- belassen. Hier möchte ich jedoch - mit aller nötigen Zurückhaltung - den Gedanken aussprechen, dass luw. -šš-/-s-vielleicht doch anders erklärt werden muss: als regelrechte Entsprechung des finn.-ugr. - $t-$ ! So kommt man schon wieder auf eine urspr. vielleicht paradigmatische Variation normal $-s-$ : kombinatorisch $-t-$.

Als Inhaber der idg. Vertretung - $t$ - des ural. - $t$ - steht jedoch *uet- nicht ganz vereinzeit da; ich mache noch auf folgende zwei sichere Fälle aufmerksam:

a) neben idg. *(a)ued- „benetzen; Wasser“ nebst der Variante idg. *ues- „feuchten, nass“ oben Pkt. IV steht ags. wabum M. „Woge“ (die Gruppe *unbiō ds. kann auch anders angereiht werden, s. Verf., Indogermanica minora I (Ljubljana 1971), 35ff.), aus idg. *uot-;

b) neben idg. *ued(h)- „führen“ oben Pkt. IV Nr. (4) steht im Germ. schon wieder eine Variante mit - $\mathrm{p}$ - = idg. *-t-: ahd. widomo, widemo „Mitgift“, mhd. wideme, widem, nhd. widmen (im Ags. mit urspr. *-d-: weotuma usw.).

In beiden letzteren Fällen steht das unregelmässige idg. * $t$-vor einem Nasalsuffix, also gerade in einer Lage, die sonst (s. Punkt IV!) idg. ${ }^{*}-d$-zur Folge hatte. Beide Fälle sind demnach recht schwierig, aus der Welt können sie aber nicht geschaffen werden.

Das idg. *uet- „Jahr“ bietet andererseits viel mehr Möglichkeiten zu einer besonderen Erklärung: vor allem ist auf die $(e) s$-Erweiterung ai. vatsá- und Verwandtes Gewicht zu legen: hier stand das sich erst entwickelnde *-p-unmittelbar vor einem weiteren b-Element $=$ später das $s$-Suffix (Kapitel II Nr. (8)!) und musste so zwar dissimiliert werden, jedoch tonlos bleiben. Ähnliches im Nom. Sg. *uet-s, im Lok. Pl. *uet-su, auch im Nom. Pl. *uet-es usw.

Obwohl nicht alle $t$-Fälle eindeutig erklärt werden konnten, so bleibt als Tatsache bestehen, das wir neben regelrechter Vertretung des ural. schwachen intervokalischen $t$-im Idg. auch kombinatorische Vertretungen $-d$ - und - $t$-finden. Sie können jedoch an der Richtigkeit der Entsprechung ural. $-t-$ $\sim$ idg. - $s$-kaum Abbruch tun.

VI. Die obige Untersuchung hat gezeigt, dass als regelrechte Entsprechungen ural. schwacher Laute - $p$ - und $-t$ - in intervokalischer Stellung im Idg. Spiranten gelten müssen. Da auch für ural. schwaches $-k$-in intervokalischer Stellung das Idg. ein $-H-=$ wahrscheinlich echtem Spirant aufweist, kann man nun folgende Regel aufstellen:

Uralischen schwachen Tenues in intervokalischer Stellung steht im Indogermanischen jeweils Spirant gegenüber. Da nun nach Pkt. II die 
uralischen Verhältnisse altertümlicher sein müssen als die indogermanischen, muss auch das Indouralische hier schwache Verschlusslaute besessen haben.

Die Spirantisierung der eben genannten schwachen Verschlusslaute in intervokalischer Stellung, die das Vorurindogermanische vollzog, ist augenscheinlich Folge einer allgemeinen Tendenz des Vorurindogermanischen, alle inlautenden Verschlusslaute in passender Stellung (zwischen Vokalen, nach Vokal und vor Konsonant, zwischen Nasal und Vokal) zu einer Lockerung der Muskelspannung $z u$ bewegen. Im allgemeinen ist daraus Folgendes herzuleiten:

a) zwischen Vokalen und nach Vokal vor Konsonant:

schwache Verschlusslaute werden im Idg. zu Spiranten;

starke (doppelte) Verschlusslaute werden im Idg. zu einfachen Tenues;

b) zwischen Nasal und Vokal:

schwache Tenues werden zu idg. Mediä aspiratä, Übergangsstufe ist eine Art von Affrikatä, bestehend aus Mediä + tönende Spiranten.

Übersichtstabelle (nur das Hauptsächliche):

\begin{tabular}{|c|c|c|c|c|}
\hline & Indoural. & Ural. & Voruridg. & Urindogermanisch \\
\hline $\begin{array}{l}\text { Schwache } \\
\text { Tenues } \\
(p, t, k)\end{array}$ & $\mathrm{p}$ & $\mathrm{p}$ & $\mathrm{p}-/-\varphi-$ & $\mathrm{p}-/-\varphi->-\mathrm{u}-$ \\
\hline $\begin{array}{l}\text { Starke } \\
\text { Tenues } \\
(\mathrm{pp}, \mathrm{tt}, \mathrm{kk})\end{array}$ & $\mathrm{tt}$ & $\mathrm{tt}$ & $-/--t(t)-$ & $\begin{array}{l}\text {-t/-tt- (heth.)*, } \\
-\mathrm{t}-, \text {,d-; auch - -? }\end{array}$ \\
\hline $\begin{array}{l}\text { Nasalgruppen } \\
(\mathrm{mp}, \mathrm{nt}, \mathrm{mt} \\
\text { nk usw.) }\end{array}$ & $\begin{array}{l}\text {-mp- } \\
\text {-nt- } \\
\text {-mt- } \\
\text {-nk- }\end{array}$ & $\begin{array}{l}\text {-mp- } \\
\text {-nt- } \\
\text {-mt- } \\
\text {-nk- }\end{array}$ & $\begin{array}{ll}-\mathrm{m} \beta- & (-) \\
-\mathrm{n} \delta- & (-) \\
-\mathrm{n} \delta- & (-) \\
-\eta \gamma- & (-)\end{array}$ & $\begin{array}{lll}\text { (m) } \mathrm{b} \beta- & / & \text {-mbh- } \\
(\mathrm{n}) \mathrm{d} \delta- & / & \text {-ndh- } \\
(\mathrm{n}) \mathrm{d} \delta- & / & \text {-ndh- }- \\
(\mathrm{n}) \mathrm{d} \delta- & / & \text {-ndh- }\end{array}$ \\
\hline
\end{tabular}

Anm.: () = Laut oder Lautzeichen kann fehlen;

I = freier Wechsel;

griechische Buchstaben = stimmhafte Spiranten.

* doppelte Tenues im Heth. noch erhalten!

Von den drei Dentalreihen ist nur eine symbolische vertreten, ebenso von den drei Gutturalreihen.

VII a) Wie in Arm. aus idg. intervokalischen - $p$ - über - $p h$-ein historisches $-v$ entsteht (vgl. Meillet. Esqu. ${ }^{2}$ 3I), so wird auch im Voruridg. aus indoural. -p-(schwache Tenuis) zunächst tonloses - $\varphi$ - (bilabialer tonloser Spirant), erst im letz- 
ten Zeitalter der uridg. Sprachentwicklung tönendes $-\boldsymbol{u}$ - (Vorstufe natürlich $-\boldsymbol{\beta}$-, bilabialer tönender Spirant). Die Dentale und Gutturale blieben hier auch in der letzten Entwicklungsphase tonlos: $-s-,-b-,-H$ - (gutturaler tonloser Spirant oder echter tonloser Laryngal?), $-d$ - als spezielle Entwicklungsrichtung ausgenommen. Auch im Arm. blieb das Endprodukt von idg. $-q-,-q_{h}^{u}$ - zwischen Vokalen tonlos: $-k h$ - (Meillet, Esqu. ${ }^{2} 29$ ) oder $-x$ - (Verf., KZ. 74 (1956), 225 unten).

b) Wie im Arm. Tenues hinter Nasal tönend wurden, ist auch hier mit ähnlichen Vorgängen zu rechnen, nur muss man m.E. zunächst an tönende Spiranten denken: $-m \beta$ - usw., erst dann unter Wirkung der Nasale die Stufe $-m b \beta$ - und daraus $-m b h$ - usw. als letztes Stadium im noch ungeteilten Indogermanisch. Vgl. Indouralica XVI = Orbis XXII (1973), Schlussfolgerungen III; auch Orbis XIX (1970), $318 \mathrm{f}$.

c) In betreff der Vertretungen der indouralischen starken Tenues und Nasalgruppen auf dem indogermanischen Gebiet kann man jedoch auch einige von denjenigen in der Tabelle verschiedene Vertretungen beobachten, die an einem anderen Ort zur Sprache kommen. Das in der Tabelle Gegebene gilt eigentlich nur für das sog. Brugmannsche Indogermanisch.

d) Bei dem beschriebenen Stand der Lautungen im Indogermanischen ist es klar, dass das Indouralische im Besitz von nur tonlosen (schwachen oder starken) Verschlusslauten war, sei es im Anlaut oder im Inlaut. Diesen Zustand bewahrte das Uralische sehr treu, während im Idg. die Tendenz zur Lockerung des Verschlusses als Folge der vokalischen bzw. sonoren Umgebung das alte System in ein ganz neues umwandelte.

\section{Schluss}

Die Ergebnisse des vorliegenden Aufsatzes können nun in folgende Sätze zusammengefasst werden:

a) uralischem schwachem - $p$ - in intervokalischer Stellung entspricht idg. - - n-;

b) uralischem schwachem - $t$ - in intervokalischer (oder postvokalischer Stellung) entspricht normal idg. $-s-$, nach Guttural - b-, nur unter besonderen Bedingungen als kombinatorische Variante auch - $d$-, selten $-t$. 
Povzetek

\section{INDO-URALICA IX}

Gre za eno izmed razprav (člankov ali monografij) iz niza Indo-Uralica I-XIX, ki imajo namen, dokončno dokazati genetično sorodnost indoevropșe in uralske jezikovne družine.

Gradivo je razporejeno po glasovno-historičnih vidikih, od najpreprostejših glasovnih odnsov do zapletenih sprememb (IU. I in II obravnavata ievr.-ural. $m, n, r, l$ in $j$, $w$, skoraj enaka na obeh straneh, IU.XIX pa ural. $\breve{s}=$ ievr. $h$, laringale, dolžine in vokalne barve itd.).

Pričujoči članek obdeluje pomembne spremembe v iver. konsonantizmu, v nasprotju z ural., kjer je prvotni status veliko bolje ohranjen. Gre za ievr. odprtje zapore, tako da ena kategorija konsonantov, iu. šibki zaporniki, med vokali in za vokali, preide v omenjenih razmerah v nezveneče pripornike. Naš članek obravnava labiale in dentale te vrste, guturali te vrste pa so obdelani v: IU. X. in objavljenim v Lingu. XXV (1985), ss. 193-262; k takemu pravilu (ievr. $-H$ - $=$ ural. $-k$-) cf. še moje Laryng. 1970 passim.

Članek za dokazovanje pravilnosti zgoraj omenjenih glasovnih pravil je podprt s koordinacijo vseh treh že v Laryng. 1970, str. 35/217 odn. ibd, str. 20/202.

Pričujočo razpravo utemeljujejo Schlussfolgerungen (Zaključki), kjer zvemo nadaljnje o:

I. Enačbi ural, $-k-=$ ievr. $-H$-Bojan Čop pred 1950 ; = laringalna teorija; ievr. ustrezniki ural. $-p$ - in $-t$ - šle kasneje; gl. $v$ tekstu.

II. Dokazi za prvotnost zaporniškega izgovora, ural. $-p-$-, $-t-$ in $-k$ - torej starejši kot ievr. odnosniki.

III. Zgornje velja tudi ob pritegnitvi zaimka 2.S. v funkciji osebila 2.Sg., 2.Du. in 2.Pl. Prvotnost tenues potrjujejo tudi altajski jeziki (kjer imamo $\mathrm{p}, \mathrm{t}, \mathrm{k}$ ).

IV. Obravnava izjem $z$ ievr. $-d$ - : ural. $-t-(7$ ievr. $d, 2$ č). Poskusi razlage $z$ disimilacijo ali delno $z$ asimilacijo.

V. Beseda za "leto“, $x_{\text {wōte. }}$

VI. Grundgesetz: Iz vsega gornjega moremo izvesti pravilo: Uralskim šibkim zapornikom $p, t, k$ ustrezajo v medvokalni legi spirantina ievr. strani, torej $\beta, \mathrm{s} / \mathrm{b}, \mathrm{x}>\mathrm{H}$. Delo zaključuje razpredelnica. 